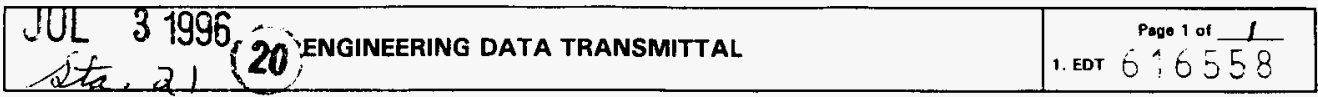

\begin{tabular}{|c|l|}
\hline $\begin{array}{l}\text { 2. To: (Receiving Organization) } \\
\text { Packaging Safety Engineering }\end{array}$ & $\begin{array}{l}\text { 3. From: coriginating } \\
\text { Criticality and }\end{array}$ \\
\hline 5. Proj./Prog./Dept./Div.: & $\begin{array}{l}\text { 6. Cog. Engr.: } \\
\text { W8M730 }\end{array}$ \\
\hline
\end{tabular}

8. Originator Renarks:

Approval/Release

4. Related EDT No.:

Shielding

616558

7. Purchase Order No.:

NA

9. Equip. /Component +No.:

NA

10. System/Bldg./Facility:

11. Receiver Remarks:

12. Major Assm. Dwg. No.:

NA

13. Permit/Permit Application No.: NA

14. Required Response Date:

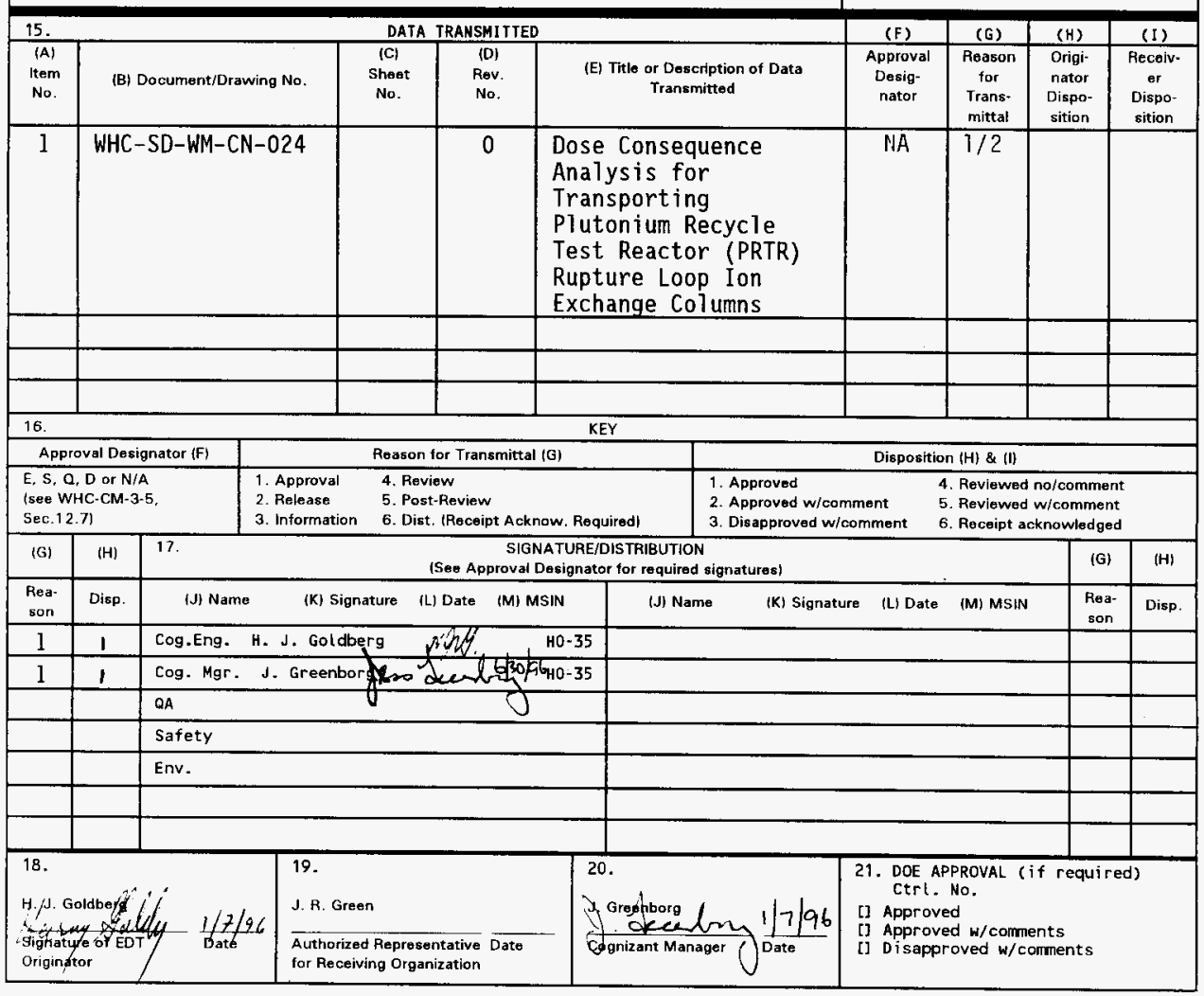




\section{Dose Consequence Analysis for Transporting Plutonium Recycle Test Reactor (PRTR) Rupture Loop lon Exchange Columns}

H. J. Goldberg

Westinghouse Hanford Company, Richland, WA 99352

U.S. Department of Energy Contract DE-AC06-87RL10930

$\begin{array}{lll}\text { EDT/ECN: } & 616558 & \text { UC: } 510 \\ \text { Org Code: } & 8 M 730 & \text { Charge Code: } \\ \text { B\&R Code: } & \text { EW31350040 } & \text { Tota1 Pages: } 37\end{array}$

Key Words: Ion Exchange Column, 309 Building, PRTR, Dose Consequences, SARP

Abstract: Ion exchange columns from the 309 Plutonium Recycle Test Reactor rupture loop must be shipped to the solid waste burial ground. The enclosed calculational note documents the calculations used to calculate the absorbed doses expected in the case of a postulated accident.

TRADEMARK DISCLAIMER. Reference herein to any specific commercial product, process, or service by trade name, trademark, manufacturer, or otherwise, does not necessarily constitute or imply its endorsement, recommendation, or favoring by the United States Government or any agency thereof or its contractors or subcontractors.

Printed in the United States of America. To obtain copies of this document, contact: WHC/BCS Document Control Services, P.O. Box 1970, Mailstop H6-08, Richland WA 99352, Phone (509) 372-2420; Fax (509) 376-4989.
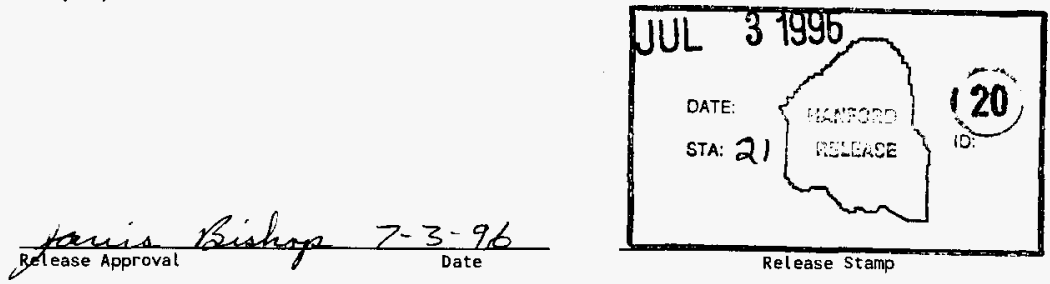

\section{Approved for Public Release}


WHC-SD-WM-CN-024

Rev. 0

Page 1 of 36

\title{
Dose Consequence Analysis for Transporting \\ Plutonium Recycle Test Reactor (PRTR) \\ Rupture Loop Ion Exchange Columns
}

\author{
Harvey Goldberg \\ May 8, 1996
}

\begin{abstract}
Purpose
This calculational note documents the calculation of absorbed doses expected for a postulated accident during shipment of Plutonium Recycle Test Reactor (PRTR) rupture loop ion exchange columns per Internal Memo 8M730-HJG-96-007 (Reference 1).
\end{abstract}

\section{Methodology}

The methodology of performing these calculations in support of the radiological dose section of an SARP is set forth in Reference 3 . It is based on the Q-system outlined in Reference 4, originally developed for calculation of $A_{1} / A_{2}$ values used in the determination of packaging requirements.

Reference 3 and this analysis use this methodology to determine dose, given an activity, rather than determining an activity, given a dose limit.

As sumptions

The following is written to facilitate inclusion into the SARP. 
WHC-SD-WM-CN-024

Rev. 0

Page 2 of 36

\subsection{CONTENTS EVALUATION}

\subsection{CHARACTERIZATION}

Twelve ion exchange (IX) columns from the 309 Plutonium Recycle Test Reactor (PRTR) rupture loop wi11 be placed in three $12.23 \mathrm{~m}^{3}$ metal boxes $\left(6^{\prime} \times 6^{\prime} \times 12^{\prime}\right)$. These columns have been designated as a non-dangerous waste in accordance with the Washington State Dangerous Waste Regulations (Ch 173-303, Washington Administrative Code). Two of the boxes (Boxes 1 \& 2) have been classified as category three and one (Box 3 ) will be category one. The void space existing after the IX columns are loaded into the boxes will be filled with grout. 
WHC-SD-WM-CN-O24

Rev. 0

Page 3 of 36

Input Data

\subsubsection{Source Term}

The following source term has been supplied by the customer (Reference 12).

\begin{tabular}{|c|c|c|c|c|c|}
\hline & Vxane I. Box & 1. $(1) \times$ coolinin & 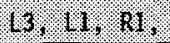 & and $\mathrm{ar} / \mathrm{s}(\mathrm{W})$ & \\
\hline Isotope & L3 & L] & $\mathrm{RI}$ & $\mathrm{R} 2$ & Total \\
\hline${ }^{241} \mathrm{Am}$ & $2.40 \mathrm{e}-01$ & & & & $2.40 \mathrm{e}-01$ \\
\hline${ }^{233} \mathrm{~Pa}$ & $2.32 \mathrm{e}-06$ & & & & $2.32 \mathrm{e}-06$ \\
\hline${ }^{237} \mathrm{~Np}$ & $2.32 \mathrm{e}-06$ & & & & $2.32 \mathrm{e}-06$ \\
\hline${ }^{234} \mathrm{U}$ & $1.51 \mathrm{e}-05$ & & & & $1.51 \mathrm{e}-05$ \\
\hline${ }^{235} \mathrm{U}$ & $6.69 e-07$ & & & & $6.69 \mathrm{e}-07$ \\
\hline${ }^{236} \mathrm{U}$ & $3.45 \mathrm{e}-07$ & & & & $3.45 e-07$ \\
\hline${ }^{238} \mathrm{U}$ & $1.55 \mathrm{e}-05$ & & & & $1.55 e-05$ \\
\hline${ }^{238} \mathrm{Pu}$ & $4.25 e-03$ & & & & $4.25 e-03$ \\
\hline${ }^{239} \mathrm{Pu}$ & $4.91 e-02$ & & & & $4.91 \mathrm{e}-02$ \\
\hline${ }^{240} \mathrm{Pu}$ & $2.22 \mathrm{e}-02$ & & & & $2.22 \mathrm{e}-02$ \\
\hline${ }^{241} \mathrm{Pu}$ & $4.53 e-01$ & & & & $4.53 e-01$ \\
\hline${ }^{242} \mathrm{Pu}$ & $6.37 e-06$ & & & & $6.37 e-06$ \\
\hline $\begin{array}{c}\text { Total } \\
\text { TRU }\end{array}$ & $7.69 e-01$ & $0.00 \mathrm{e}+00$ & $0.00 e+00$ & $0.00 \mathrm{e}+00$ & $7.69 \mathrm{e}-01$ \\
\hline${ }^{137} \mathrm{Cs}$ & $2.10 \mathrm{e}+00$ & $4.30 \mathrm{e}-03$ & $1.01 \mathrm{e}-02$ & $4.67 e-01$ & $2.58 e+00$ \\
\hline${ }^{137 m} \mathrm{Ba}$ & $1.99 \mathrm{e}+00$ & $4.07 e-03$ & $9.55 \mathrm{e}-03$ & $4.42 \mathrm{e}-01$ & $2.45 e+00$ \\
\hline${ }^{90} \mathrm{Sr}$ & $3.40 \mathrm{e}-01$ & $6.90 \mathrm{e}-04$ & $1.60 \mathrm{e}-03$ & $7.50 e-02$ & $4.17 \mathrm{e}-01$ \\
\hline${ }^{90} Y$ & $3.40 \mathrm{e}-01$ & $6.90 \mathrm{e}-04$ & $1.60 \mathrm{e}-03$ & $7.50 \mathrm{e}-02$ & $4.17 e-01$ \\
\hline${ }^{60} \mathrm{Co}$ & $1.33 e-04$ & $4.20 \mathrm{e}-05$ & $8.19 \mathrm{e}-05$ & $7.52 e-04$ & $1.01 \mathrm{e}-03$ \\
\hline Total & $5.54 e+00$ & $9.79 e-03$ & $2.29 \mathrm{e}-02$ & $1.06 \mathrm{e}+00$ & $6.63 e+00$ \\
\hline
\end{tabular}


WHC-SD-WM-CN-024

Rev. 0

Page 4 of 36

\begin{tabular}{|c|c|c|c|c|c|}
\hline & $12,314.2 .60 x$ & (IX & Q6. $.12,12$ & $010.14)(4)$ & 4 \\
\hline Isotope & R3 & $\mathrm{C} 2$ & L2 & L4 & Total \\
\hline${ }^{241} \mathrm{Am}$ & $3.23 e-02$ & $1.72 e-01$ & & $7.54 \mathrm{e}-02$ & $2.80 \mathrm{e}-01$ \\
\hline${ }^{233} \mathrm{~Pa}$ & $3.13 \mathrm{e}-07$ & $1.67 e-06$ & & $7.31 \mathrm{e}-07$ & $2.71 e-06$ \\
\hline${ }^{237} \mathrm{~Np}$ & $3.13 \mathrm{e}-07$ & $1.67 \mathrm{e}-06$ & & $7.31 \mathrm{e}-07$ & $2.71 e-06$ \\
\hline${ }^{234} \mathrm{U}$ & $2.04 e-06$ & $1.09 \mathrm{e}-05$ & & $4.76 e-06$ & $1.77 \mathrm{e}-05$ \\
\hline${ }^{235} U$ & $9.01 \mathrm{e}-08$ & $4.81 \mathrm{e}-07$ & & $2.10 e-07$ & $7.81 \mathrm{e}-07$ \\
\hline${ }^{236} \mathrm{u}$ & $4.65 e-08$ & $2.48 \mathrm{e}-07$ & & $1.09 \mathrm{e}-07$ & $4.04 e-07$ \\
\hline${ }^{238} U$ & $2.09 \mathrm{e}-06$ & $1.12 \mathrm{e}-05$ & & $4.88 e-06$ & $1.82 \mathrm{e}-05$ \\
\hline${ }^{238} \mathrm{Pu}$ & $5.73 e-04$ & $3.06 e-03$ & & $1.34 \epsilon-03$ & $4.97 e-03$ \\
\hline${ }^{239} \mathrm{Pu}$ & $6.62 \mathrm{e}-03$ & $3.53 e-02$ & & $1.55 \mathrm{e}-02$ & $5.74 e-02$ \\
\hline${ }^{240} \mathrm{Pu}$ & $2.99 e-03$ & $1.60 \mathrm{e}-02$ & & $6.99 e-03$ & $2.60 \mathrm{e}-02$ \\
\hline${ }^{241} \mathrm{Pu}$ & $6.11 e-02$ & $3.26 e-01$ & & $1.43 e-01$ & $5.30 e-01$ \\
\hline${ }^{242} \mathrm{Pu}$ & $8.59 e-07$ & $4.58 \mathrm{e}-06$ & & $2.00 \mathrm{e}-06$ & $7.44 e-06$ \\
\hline $\begin{array}{c}\text { Tota } 1 \\
\text { TRU }\end{array}$ & $1.04 e-01$ & $5.52 e-01$ & $0.00 \mathrm{e}+00$ & $2.42 e-01$ & $8.98 e-01$ \\
\hline${ }^{137} \mathrm{Cs}$ & $1.10 \mathrm{e}+00$ & $3.35 e-03$ & $1.36 \mathrm{e}-01$ & $1.08 \mathrm{e}-02$ & $1.25 e+00$ \\
\hline${ }^{137} \mathrm{Ba}$ & $1.04 e+00$ & $3.17 e-03$ & $1.29 \mathrm{e}-01$ & $1.02 \mathrm{e}-02$ & $1.18 \mathrm{e}+00$ \\
\hline${ }^{90} \mathrm{Sr}$ & $1.80 e-01$ & $5.40 e-04$ & $2.20 \mathrm{e}-02$ & $1.70 \mathrm{e}-03$ & $2.04 e-01$ \\
\hline${ }^{90} \mathrm{Y}$ & $1.80 \mathrm{e}-01$ & $5.40 \mathrm{e}-04$ & $2.20 \mathrm{e}-02$ & $1.70 \mathrm{e}-03$ & $2.04 \mathrm{e}-01$ \\
\hline${ }^{60} \mathrm{Co}$ & $1.57 \mathrm{e}-03$ & $8.94 e-05$ & $1.73 \mathrm{e}-04$ & $1.24 \mathrm{e}-04$ & $1.96 \mathrm{e}-03$ \\
\hline Total & $2.61 \mathrm{e}+00$ & $5.60 \mathrm{e}-01$ & $3.09 \mathrm{e}-01$ & $2.67 \mathrm{e}-01$ & $3.74 \mathrm{e}+00$ \\
\hline
\end{tabular}


WHC-SD-WM-CN-024

Rev. 0

Page 5 of 36

\begin{tabular}{|c|c|c|c|c|c|}
\hline \multicolumn{2}{|c|}{ Table $3.80 \%$ ? } & $60111 m$ ms & 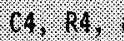 & X.ter. & 1. \\
\hline Isotope & C3 & C4 & R4 & R5/RLIXI & Total \\
\hline${ }^{241} \mathrm{Am}$ & & & & & $0.00 \mathrm{e}+00$ \\
\hline${ }^{233} \mathrm{~Pa}$ & & & & & $0.00 e+00$ \\
\hline${ }^{237} \mathrm{~Np}$ & & & & & $0.00 \mathrm{e}+00$ \\
\hline${ }^{234} U$ & & & & & $0.00 \mathrm{e}+00$ \\
\hline${ }^{235} \mathrm{U}$ & & & & & $0.00 \mathrm{e}+00$ \\
\hline${ }^{236} \mathrm{U}$ & & & & & $0.00 \mathrm{e}+00$ \\
\hline${ }^{238} \mathrm{U}$ & & & & & $0.00 \mathrm{e}+00$ \\
\hline${ }^{238} \mathrm{Pu}$ & & & & & $0.00 \mathrm{e}+00$ \\
\hline${ }^{239} \mathrm{Pu}$ & & & & & $0.00 e+00$ \\
\hline${ }^{240} \mathrm{Pu}$ & & & & & $0.00 e+00$ \\
\hline${ }^{241} \mathrm{Pu}$ & & & & & $0.00 \mathrm{e}+00$ \\
\hline${ }^{242} \mathrm{Pu}$ & & & & & $0.00 \mathrm{e}+00$ \\
\hline $\begin{array}{c}\text { Total } \\
\text { TRU }\end{array}$ & $0.00 \mathrm{e}+00$ & $0.00 \mathrm{e}+00$ & $0.00 \mathrm{e}+00$ & $0.00 \mathrm{e}+00$ & $0.00 \mathrm{e}+00$ \\
\hline${ }^{137} \mathrm{Cs}$ & $1.36 \mathrm{e}-09$ & $1.36 \mathrm{e}-09$ & $3.76 \mathrm{e}-02$ & & $3.76 \mathrm{e}-02$ \\
\hline${ }^{137 m} \mathrm{Ba}$ & $1.29 \mathrm{e}-09$ & $1.29 \mathrm{e}-09$ & $3.56 \mathrm{e}-02$ & & $3.56 \mathrm{e}-02$ \\
\hline${ }^{90} \mathrm{Sr}$ & $2.20 \mathrm{e}-10$ & $2.20 \mathrm{e}-10$ & $6.00 \mathrm{e}-03$ & & $6.00 \mathrm{e}-03$ \\
\hline${ }^{90} Y$ & $2.20 \mathrm{e}-10$ & $2.20 \mathrm{e}-10$ & $6.00 \mathrm{e}-03$ & & $6.00 \mathrm{e}-03$ \\
\hline${ }^{60} \mathrm{CO}$ & $1.55 \mathrm{e}-08$ & $1.55 e-08$ & $5.32 e-04$ & $7.70 \mathrm{e}-05$ & $6.09 \mathrm{e}-04$ \\
\hline Total & $1.86 e-08$ & $1.86 \mathrm{e}-08$ & $8.58 \mathrm{e}-02$ & $7.70 \mathrm{e}-05$ & $8.58 \mathrm{e}-02$ \\
\hline
\end{tabular}

The actinides have not been decayed. However, the ${ }^{90} \mathrm{Sr}$ and ${ }^{137} \mathrm{Cs}$ have had their respective daughter products added and the short lives isotope ${ }^{233} \mathrm{~Pa}$ has had its precursor, ${ }^{237} \mathrm{~Np}$ added. Note that TRU stands for the english word transuranic. In regulatory parlance the acronym TRU only includes those transuranic isotopes with halflives of 20 years or more and occasionally includes some non-transuranic elements. 
WHC-SD-WM-CN-024

Rev. 0

Page 6 of 36

\section{Calculations}

\subsection{DOSE CONSEQUENCES OF ACCIDENTS}

\subsection{INTRODUCTION}

It has been assumed that the IX columns undergo an accident in which the enclosing box is completely compromised and one of the columns is broken open. The IX columns are cylinders of Amberlite or Duolite enclosed in a $1 / 8^{\prime \prime}$ steel enclosure. No credit was taken for the enclosure, i.e. the filter medium was assumed to be completely exposed.

Only one cylinder, the worst case cylinder (L3) was analyzed so as to provide a worst case situation which would be an upper bound on any other situation. As a more conservative scenario, the dose calculation of the dose from Bparticles and photons assumes an accident where the entire worst case box (four columns) is completely released. Although the material is not flammable, an engulfing fire was analyzed as well as the non-fire situation.

The methodology is based on the IAEA Q-system (Reference 4) outlined in Reference 3 and then amended (Reference 12). The IAEA Q-system was developed as an all-encompassing generalized methodology using only the isotope as the defining variable. In this document, the specifics of the package are considered. Some of the pathways for dose may be considered as not credible and, although these pathways may be covered in the IAEA guide, they are disregarded in the analysis.

\subsubsection{RESULTS}

\subsubsection{Photon Dose}

In the chosen scenario the source is modeled as a point source. No credit is taken for any self-shielding by the source. The entire contents of one of the boxes is assumed to be completely released from its steel enclosure. While only one column has been assumed to be compromised, the onsite receptor is exposed to photons from the other undamaged cylinders also. Although the steel enclosures of these cylinders will decrease this exposure, no credit was taken for this diminution of the dose.

Following the assumptions of Reference 4 it was assumed that the worker stood one meter from the accident for one half of an hour. In the fire scenario the conditions remain the same, despite the fact that the conflagration would tend to strongly discourage a worker from standing within one meter of the source for this amount of time. 
WHC-SD-WM-CN-024

Rev. 0

Page 7 of 36

The computer code ISOSHLD (References 5, 6, and 7) was used to calculate the dose rate one meter from the source. Bremsstrahlung from the B-particle. emitting radionuclides was accounted for. The fluence-to-dose conversion factors used in the calculation were the anterior-to-posterior irradiation pattern as presented in Reference 8 . The receptor is expected to receive $4.0 \times 10^{-3} \mathrm{~Sv}\left(4.0 \times 10^{-1} \mathrm{rem}\right)$.

\subsubsection{B-Particle Dose}

The IAEA methodology (Reference 4) assumes a point source of the material for this case also. As with the photon analysis, it has been assumed in this case that an entire box is completely released. The worst case box is box 1 , containing $I X$ columns $L 1, L 3, R 1$, and $R 2$. Due to the 1 imited range of $B-$ particles as compared to photons, a shielding factor due to residual shielding from material such as package debris is used by the IAEA and is also adopted here. The variation of shielding factor as a function of the maximum energy of the B-particle is graphed in Reference 4. Except for this factor no effort is made to account for either self shielding or shielding from an accurate model of the damaged package. A one half hour stay time is again assumed.

The two graphs in Reference 4 have been incorporated into a spreadsheet in order to facilitate the calculational process. The total B-ray dose was calculated as $4.4 \times 10^{-2} \mathrm{~Sv}\left(4.4 \times 10^{+0} \mathrm{rem}\right)$. Of course, this is a skin dose which, by itself is counted as an organ dose, and when being included in the $E D E$ is multiplied by the tissue weighting factor for skin $(0.01)$. As expected, the majority of the B-ray dose was due to ${ }^{90} \mathrm{Y}$.

\subsubsection{Airborne Inhalation Dose}

The computer code GXQ (Reference 9) was used to calculate the $\Psi / Q$ s for the distances and directions for the nearest site boundary. The meteorological data used were the 1983 to 1991 averages for ground (actually 10m) releases in the 200 and 300 areas. For the onsite receptor a constant distance between the release point and the receptor of $100 \mathrm{~m}$ was used. Since the 309 building is not a public exclusion area, the distance from the release to the receptor was also assumed to be $100 \mathrm{~m}$. A value for $\Psi / Q$ was chosen which was not exceeded more than $0.5 \%$ of the time using the NRC methodology.

The worst case of these was then chosen for each receptor and used in this analysis. Since it has been assumed that an accident would lead to the confiscation of any foodstuffs found to be contaminated, ingestion by the public was not considered. The worst case for both cases was $100 \mathrm{~m}$ to the north of the release in the 300 area. The $\Psi / Q$ value used was $4.21 \times 10^{-2}$ $\sec / \mathrm{m}^{3}$. 
WHC-SD-WM-CN-024

Rev. 0

Page 8 of 36

The computer code GENII (Reference 10) was then used to calculate the dose to the affected individual. The following libraries were accessed;

GENII Default Parameter Values (28-Mar-90 RAP)

Radionuclide Library - Times<100 years (23-July-93 PDR)

PNL Food Transfer Factor Library (by Z, with Fr\&0s 7/19/93 PDR)

Bioaccumulation Factor Library - (30-Aug-88 RAP)

External Dose Factors for GENII in person Sv/yr per Bq/n (8-May-90 RAP)

Worst-Case Solubilities, Yearly Dose Increments (23-Jul-93 PDR)

For the public receptors, the dose is due to the inhalation pathway alone. In order to compensate for the fact that the onsite dose was calculated at a

source-to-receptor distance of $100 \mathrm{~m}$, this dose was multiplied by a factor of thirty as recommended in Reference 3.

The analysis assumes that $100 \%$ of the inventory of the worst case column (L3) will be released in the accident. What fraction of this term is suspended in the air in respirable particles is quite a problem. Mishima's review of the published data (Reference 11) was used as the source of these data. For the non-fire case the value in Mishima for the free-fall drop of an enclosed HEPA filter onto a hard unyielding surface was chosen.

While these filters are not HEPA filters, it was felt that this value would be a bounding value which would not be exceeded in this case. In a HEPA filter the contamination is deposited on the surface of the filter medium, while for an ion exchange filter medium the contamination is adsorbed onto the medium. The release should not exceed that of a HEPA filter. Mishima gives an upper bound of $5 \times 10^{-4}$ for the airborne release fraction (ARF), and 1.0 for the respirable fraction (RF). Thus the respirable airborne release fraction (RARF) woutd be $5 \times 10^{-4}$.

Mishima has also reviewed burning of ion exchange resins. These columns use a clay medium rather than a resin, however these data were used for the present situation. Only three experiments were performed yielding a set so restricted that a medium value and spread are not meaningful concepts. However, Hanford. has made a practice of choosing the upper bound, which in this case is ARF = $7.8 \times 10^{-3}$ and $R F=9 \times 10^{-1}$, yielding a RARF of $7.0 \times 10^{-3}$. This is the value that was used in this analysis. 


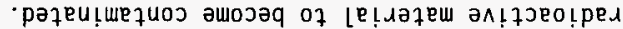

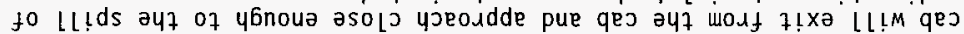

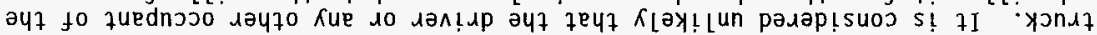

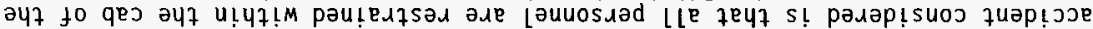

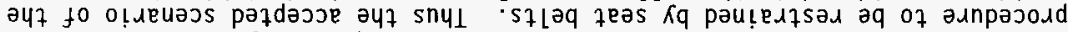

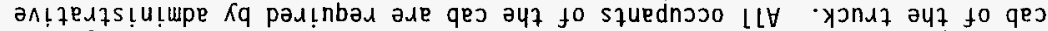

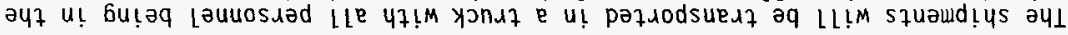

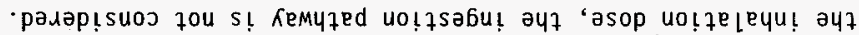

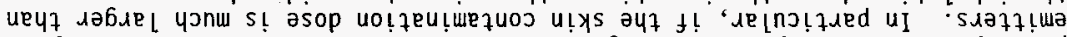

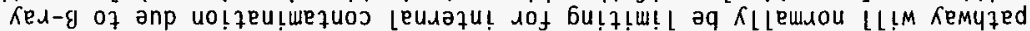
uo!fe

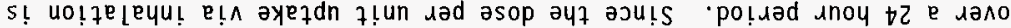

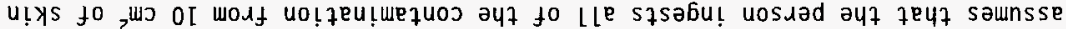

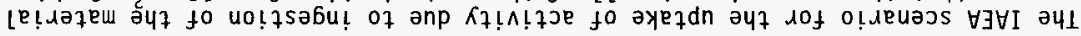

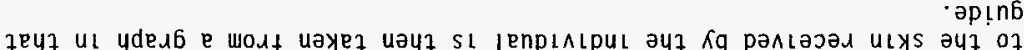

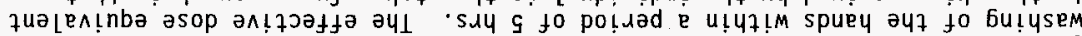

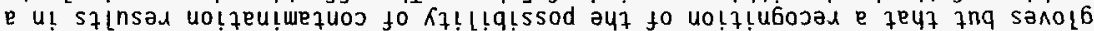

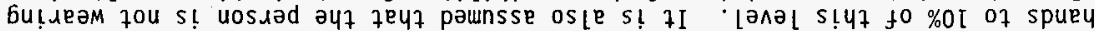

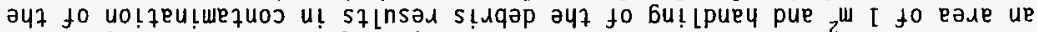

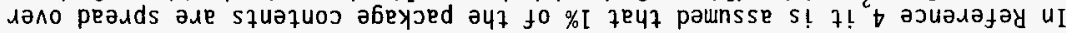

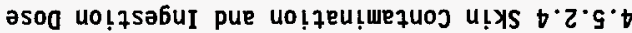

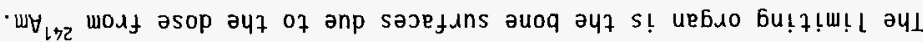

\begin{tabular}{|c|c|c|c|c|}
\hline 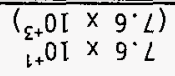 & 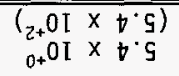 & 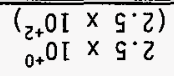 & $\begin{array}{llll}(1+0 I & \times & 8^{\circ} & 1 \\
1-0 I & \times & 8^{\circ} & 1\end{array}$ & asog uebuo \\
\hline 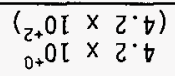 & $\begin{array}{c}\left(1+0 I \times 0_{1} \times \sigma^{\circ}\right) \\
1.0\left[\times 0^{\circ} \varepsilon\right.\end{array}$ & $\begin{array}{cccc}(l+0 I & x & t & I) \\
l-O I & x & t & I\end{array}$ & 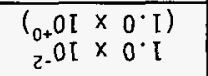 & 303 \\
\hline 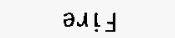 & $\partial \Lambda !\rfloor$ ON & $\partial\rfloor$ & $\partial u !\lrcorner$ ON & \\
\hline \multicolumn{2}{|c|}{ ə7!suo } & \multicolumn{2}{|c|}{ o lqnd } & \\
\hline & 16. & 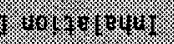 & 2. & \\
\hline
\end{tabular}

-

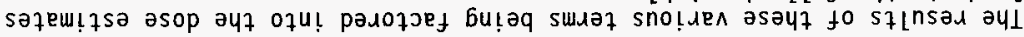

$9 \varepsilon$ to 6 ә6ed

$0 \cdot \wedge$ วу

t2O-N3-WM-OS-3HM 
WHC-SD-WM-CN-024

Rev. 0

Page 10 of 36

Also, being in the enclosed cab during the accident would render it extremely unlikely that the material would be spilled over them in the accident.

However, if the above assumptions do not hold for any particular shipment, this route of exposure will have to be evaluated.

\subsubsection{Gaseous Vapor Dose}

This term is due to submersion in a cloud of gaseous isotopes which do not become incorporated into the body. A rapid $100 \%$ release of the contents of the package is assumed. The description of this pathway in the IAEA guide concentrates entirely on releases within confining structures. No guidance is given for outside releases. However, since it has been assumed that the package considered in this document has nonexistent or negligible concentrations of these gases, the problem is moot.

This route for this particular package is not significant as there are no gaseous isotopes trapped in the material and the production rate is trivial.

\subsubsection{Neutron Dose}

The amount of TRU material is so low that neutron emissions are not of concern in this shipment. In addition to spontaneous and induced fission neutrons, neutrons from the $(\alpha, n)$ reaction were considered and dismissed as negligible.

\subsubsection{Special Considerations}

Alpha particle emitters are not of significance in the material considered in this document, neither are tritium nor radon. The alpha particle emitters are of a low concentration and their effect will be through the mechanism of inhalation and ingestion. These effects have been considered separately. Bremsstrahlung has been included in the consideration of photon effects and the effects of short lived daughter products have been included in all of the calculations. Where these isotopes were significant they were assumed to be in equilibrium with their longer lived parent isotopes. 
WHC-SD-WM-CN-024

Rev. 0

Page 11 of 36

Results

\subsubsection{Total Dose}

The results of these analyses, although reported with two significant figures, can only be trusted to one. For this reason, the highest of the doses are generally chosen except when two or more dose pathways yield comparable results, in which case they are added. However, the inhalation results are the limiting case.

\begin{tabular}{|c|c|c|c|c|}
\hline & \multicolumn{2}{|c|}{ Public } & \multicolumn{2}{|c|}{ Onsite } \\
\hline & No Fire & Fire & No Fire & Fire \\
\hline $\mathrm{EDE}$ & $\begin{array}{c}1.0 \times 10^{-2} \\
\left(1.0 \times 10^{+0}\right)\end{array}$ & $\begin{array}{r}1.4 \times 10^{-1} \\
\left(1.4 \times 10^{+1}\right)\end{array}$ & $\begin{array}{c}3.0 \times 10^{-1} \\
\left(3.0 \times 10^{+1}\right)\end{array}$ & $\begin{array}{r}4.2 \times 10^{+0} \\
\left(4.2 \times 10^{+2}\right)\end{array}$ \\
\hline Borgang Dorfe & $\begin{array}{l}1.8 \times 10^{-1} \\
\left(1.8 \times 10^{+1}\right)\end{array}$ & $\begin{array}{r}2.5 \times 10^{+0} \\
\left(2.5 \times 10^{+2}\right)\end{array}$ & $\begin{array}{c}5.4 \times 10^{+0} \\
\left(5.4 \times 10^{+2}\right)\end{array}$ & $\begin{array}{r}7.6 \times 10^{+1} \\
\left(7.6 \times 10^{+3}\right)\end{array}$ \\
\hline
\end{tabular}

Conclusions

No conclusions can be drawn from this analysis. The allowable limits on packages used in shipping are variable depending on the expected frequency of the accident under consideration. The determination of conformity to the limits is left to the customer. 
WHC-SD-WM-CN-024

Rev. 0

Page 12 of 36

\section{REFERENCES}

1) Internal Memo 8M730-HJG-96-007, 309 PRTR Rupture Loop IX Columns Shipping Boxes Source Term and Accident Analysis, H. J. Goldberg to J. R. Green, 23 April 1996

2) Safety Analysis and Nuclear Engineering Work Procedure, WHC-CM-6-32, Calculation Note WP-6.x, to be issued

3) Report on Equivalent Safety for Transportation and Packaging of Radioactive Materials, WHC-SD-TP-RPT-001, Rev. 0, dated December 1993.

4) Explanatory Material for the IAEA Regulations for the Safe Transport of Radioactive Material, IAEA Safety Series No. 7, 1985 Edition as amended in 1990 .

5) ISOSHLD- A Computer Code for General Purpose Isotope Shielding Analysis, BNWL-236, R. L. Enge1, J. Greenborg, M. M. Hendrickson, dated June 1966.

6) ISOSHLD-II: Code Revision to Include Calculation of Dose Rate from Shielded Bremsstrahlung Sources, G. L. Simmons, J. J. Regimbal, J. Greenborg, E. L. Kelly, Jr., H. H. van Tuyl, dated March 1967.

7) ISO-PC Version 1.98 - User's Guide, WHC-SD-WM-UM-030, Rev 0, P. D. Rittmann, dated May 1995.

8) Neutron and Gamma-ray Fluence-to-dose Factors, ANSI/ANS-6.1.1-1991.

9) GXQ 3.1 Users' Guide, WHC-SD-GN-SWD-30002, Rev.0, B. E. Hey, dated June 1993.

10) GENII- The Hanford Environmental Dosimetry Software System, PNL-6584, B. A. Napier, D. L. Strenge, R. A. Peloquin, J. V. Ramsde11, dated November 1988.

11) Recommended Values and Technical Bases for Airborne Release Fractions (ARFs), Airborne Release Rates (ARRs), and Respirable Fractions (RFs) at DOE Non-Reactor Nuclear Facilities, Draft, DOE-HDBK-0013-93, J. Mishima.

12) Janet Green, 1996, Private Communication 
Computer Output

GXQ Version 3.1 B June 8,1993

\begin{abstract}
General Purpose Atmospheric Dispersion Code Produced by Radiological \& Toxicological Analysis Westinghouse Hanford Company

Users Guide documented in WHC-SD-GN-SWD-3002 Rev. 0. Validation documented in WHC-SD-GN-SWD-3003 Rev. 0. Code Custodian is Brit E. Hey, WHC, ext. 6-2921.
\end{abstract}

Run Date $=5 / 8 / 1995$

Run Time $=13: 1811.67$

\title{
INPUT ECHO:
}

300 Area - Worker

c GXQ Ver. 3.1 Input File

c mode

1

C

c MODE CHOICE:

$c$ mode $=1$ then $X / Q$ based on Hanford site specific meteorology

$c$ mode $=2$ then $X / Q$ based on atmospheric stability class and wind speed

c mode $=3$ then $X / Q$ plot file is created

C

c LOGICAL CHOICES:

c ifox inorm icdf ichk isite ipop icon

$c$ ifox $=t$ then joint frequency used to compute frequency to exceed $X / Q$

$c=f$ then joint frequency used to compute annual average $X / Q$

$c$ inorm $=t$ then joint frequency data is normalized (as in GENII)

$c=f$ then joint frequency data is un-normalized

$c$ icdf $=t$ then cumulative distribution file created (CDF.OUT)

c $=f$ then no cumulative distribution file created

$c$ ichk $=t$ then $X / Q$ parameter print option turned on

$c=f$ then no parameter print

$c$ isite $=t$ then $X / Q$ based on joint frequency data for all 16 sectors

$c=f$ then $X / Q$ based on joint frequency data of individual sectors

$c$ ipop $=t$ then $X / Q$ is population weighted

$c=f$ then no population weighting

$c$ icon $=t$ then $X / Q$ is air concentration

$c=f$ then $X / Q$ is integrated exposure 
WHC-SD-WM-CN-024

Rev. 0

Page 14 of 36

c MODEL CHOICES:

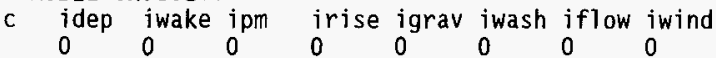

c idep $=1$ then plume depletion model turned on (Chamberlain model)

c iwake $=1$ then NRC RG 1.145 building wake model turned on

$c=2$ then MACCS virtual distance building wake model turned on

c $\mathrm{ipm}=1$ then NRC RG 1.145 plume meander model turned on

$c \quad=2$ then 5 th Power Law plume meander model turned on

c $\quad=3$ then sector average model turned on

c irise $=1$ then momentum/buoyancy plume rise model turned on, buoyancy rise based on sensible heat emission

$=2$ then momentum/buoyancy plume rise model turned on, buoyancy rise based on initial plume density

c igrav = l then gravitational settling model turned on

c iwash $=1$ then stack downwash model turned on

c iflow $=1$ then sigmas adjusted for volume flow rate

c iwind $=1$ then wind speed corrected for plume height

$c=0$ to turn any of the above models off

$\mathrm{C}$

c PARAMETER INPUT:

\begin{tabular}{|c|c|c|c|c|}
\hline $\begin{array}{l}\text { stack } \\
\text { release } \\
\text { height } \\
\text { (m) } \\
0.00000 E+00\end{array}$ & $\begin{array}{l}\text { wind } \\
\text { speed } \\
\text { height } \\
\text { (m) } \\
1.00000 E+01\end{array}$ & $\begin{array}{l}\text { mixing } \\
\text { height } \\
\text { (m) } \\
1.00000 E+03\end{array}$ & $\begin{array}{l}\text { frequency } \\
\text { to } \\
\text { exceed } \\
(\%) \\
5.00000 E-01\end{array}$ & $\begin{array}{l}\text { scaling } \\
\text { factor } \\
\text { (?) } \\
1.00000 E+00\end{array}$ \\
\hline $\begin{array}{l}\text { building } \\
\text { width } \\
\text { (m) } \\
5.00000 \mathrm{E}+00\end{array}$ & $\begin{array}{l}\text { building } \\
\text { height } \\
\text { (m) } \\
5.00000 E+00\end{array}$ & $\begin{array}{l}\text { release } \\
\text { duration } \\
\text { (hr) } \\
1.00000 E+00\end{array}$ & $\begin{array}{l}\text { deposition } \\
\text { velocity } \\
\text { (m/s) } \\
1.00000 \text { E-03 }\end{array}$ & $\begin{array}{l}\text { gravitational } \\
\text { settling } \\
\text { velocity } \\
\text { (m/s) } \\
1.00000 \mathrm{E}-03\end{array}$ \\
\hline $\begin{array}{l}\text { initial } \\
\text { plume } \\
\text { density } \\
\text { (g/cc) } \\
1.22000 E-03\end{array}$ & $\begin{array}{l}\text { initial } \\
\text { plume } \\
\text { flow } \\
\text { rate } \\
(\mathrm{m} 3 / \mathrm{s}) \\
0.00000 \mathrm{E}+00\end{array}$ & $\begin{array}{l}\text { stack } \\
\text { diameter } \\
(\mathrm{m}) \\
1.00000 \mathrm{E}+00\end{array}$ & $\begin{array}{l}\text { sensible } \\
\text { heat } \\
\text { emission } \\
\text { rate } \\
(\mathrm{ca} 1 / \mathrm{s}) \\
4.18000 \mathrm{E}+05\end{array}$ & \\
\hline
\end{tabular}

RECEPTOR DEPENDENT DATA

FOR MODE make

1 (site specific)

RECEPTOR DEPENDENT DATA

sector distance $z$-height

2 (by class \& wind speed) class windspeed distance offset $z$-height

3 (create plot file) class windspeed xmax imax ymax jmax xqmin power 
C RECEPTOR PARAMETER DESCRIPTION:

C sector $=0,1,2 \ldots($ al1, S, SSW, etc. $)$

c distance $=$ meters

C class $=1,2,3,4,5,6,7$ (P-G stability class $A, B, C, D, E, F, G)$

c windspeed $=\mathrm{m} / \mathrm{s}$

c offset = meters offset from plume centerline

c $x \max =$ maximum distance to plot or calculate to (m)

c $i \max =$ distance intervals

c $y \max =$ maximum offset to plot $(m)$

c jmax $=$ offset intervals

c xqmin $=$ minimum scaled $X / Q$ to calculate

$c$ power $=$ exponent in power function step size

MODE :

Site specific $X / Q$ calculated

LOGICAL CHOICES:

Joint frequency used to calculate $X / Q$ based on

frequency of exceedance.

No normalization of joint frequency.

$X / Q$ calculated for single sector.

Output is atmospheric dispersion coefficient.

MODELS SELECTED:

WARNING/ERROR MESSAGES:

WARNING \#3 - Scaled $X / Q$ units (shown as $\mathrm{s} / \mathrm{m} 3$ or $1 / \mathrm{m} 3$ below) do not reflect the user specified scaling factor.

JOINT FREQUENCY DATA:

300 AREA - 10 M - Pasquil1 A - G (1983 - 1991 Average)

Created $8 / 26 / 92 \mathrm{KR}$ 
WHC-SD-WM-CN-024

Rev. 0

Page 16 of 36

300 Area - Worker

\begin{tabular}{|c|c|c|c|c|c|c|c|c|}
\hline SECTOR & $\begin{array}{l}\text { DISTANCE } \\
(\mathrm{m})\end{array}$ & $\begin{array}{c}\text { RECEPT } \\
\text { HEIGHT } \\
(m)\end{array}$ & $\begin{array}{c}\text { SECT. } \\
\text { FREQ. } \\
(\%)\end{array}$ & POPULATION & $\begin{array}{c}\text { TOTAL } \\
\text { POPULATION } \\
\text { SCALED } \\
X / Q \\
(\mathrm{~s} / \mathrm{m} 3)\end{array}$ & $\begin{array}{c}\text { AVERAGE } \\
\text { INDIVIDUAL } \\
\text { SCALED } \\
X / Q \\
(s / \mathrm{m} 3)\end{array}$ & $\begin{array}{l}\text { ATM. } \\
\text { STAB. } \\
\text { CLASS }\end{array}$ & $\begin{array}{l}\text { WIND } \\
\text { SPEED } \\
(\mathrm{m} / \mathrm{s})\end{array}$ \\
\hline $\bar{S}$ & 100 & 0 & 11.55 & $\overline{1}$ & $3 . \overline{18 E-02}$ & $3.18 \mathrm{E}-02$ & $\bar{F}$ & .89 \\
\hline SSW & 100 & 0 & 5.07 & 1 & $1.16 \mathrm{E}-02$ & $1.16 \mathrm{E}-02$ & $\mathrm{~F}$ & 2.65 \\
\hline SW & 100 & 0 & 2.39 & 1 & $4.39 E-03$ & $4.39 \mathrm{E}-03$ & $\mathbf{F}$ & 7.15 \\
\hline WSW & 100 & 0 & 1.82 & l & $3.88 \mathrm{E}-03$ & $3.88 \mathrm{E}-03$ & C & .89 \\
\hline W & 100 & 0 & 4.20 & 1 & $1.51 E-02$ & $1.51 \mathrm{E}-02$ & $E$ & .89 \\
\hline WNW & 100 & 0 & 6.49 & 1 & $2.90 \mathrm{E}-02$ & $2.90 \mathrm{E}-02$ & $\bar{G}$ & 2.65 \\
\hline NW & 100 & 0 & 8.60 & 1 & $3.28 \mathrm{E}-02$ & $3.28 \mathrm{E}-02$ & $\mathrm{~F}$ & .89 \\
\hline NNW & 100 & 0 & 5.97 & 1 & $3.29 E-02$ & $3.29 \mathrm{E}-02$ & $\mathrm{~F}$ & .89 \\
\hline N & 100 & 0 & 8.76 & 1 & $4.21 E-02$ & $4.21 \mathrm{E}-02$ & F & .89 \\
\hline NNE & 100 & 0 & 8.50 & 1 & $2.89 E-02$ & $2.89 \mathrm{E}-02$ & G & 2.65 \\
\hline NE & 100 & 0 & 9.64 & 1 & $2.93 \mathrm{E}-02$ & $2.93 \mathrm{E}-02$ & G & 2.65 \\
\hline ENE & 100 & 0 & 6.13 & 1 & $1.60 \mathrm{E}-02$ & $1.60 \mathrm{E}-02$ & G & 4.70 \\
\hline & 100 & 0 & 4. & 1 & $3.16 E-02$ & $3.16 \mathrm{E}-02$ & $\mathrm{~F}$ & .89 \\
\hline & 100 & 0 & 2.8 & 1 & 2.99 & $2.99 \mathrm{E}-02$ & G & 2.65 \\
\hline & 10 & 0 & & 1 & 3.16 & $3.16 \mathrm{E}-02$ & $\mathrm{~F}$ & .89 \\
\hline SSE & 100 & 0 & 7.95 & 1 & $3.23 \mathrm{E}-02$ & $3.23 \mathrm{E}-02$ & $F$ & .89 \\
\hline
\end{tabular}

Execution Time $=0 \mathrm{hr} 0 \mathrm{~min} 1.05 \mathrm{sec}$

Stop - Program terminated. 
WHC-SD-WM-CN-024

Rev. 0

Page 17 of 36

\section{GENII Dose Calculation Program}

(Version 1.485 3-Dec-90)

Case title: Ion Exchange Column L3 - Worker

Executed on: $03 / 22 / 96$ at $11: 25: 14$

Page A. 1

This is a far-field (wide-scale release, multiple site) scenario.

Release is acute

Individual dose

THE FOLLOWING TRANSPORT MODES ARE CONSIDERED

Air

THE FOLLOWING EXPOSURE PATHS ARE CONSIDERED:

Infinite plume, external

Inhalation uptake

THE FOLLOWING TIMES ARE USED:

Intake ends after $(y r)$ : $\quad 1.0$

Dose calculations ends after (yr): $\quad 50.0$

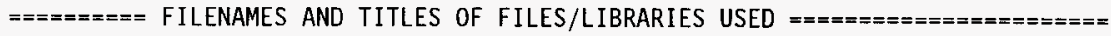

Input file name: \GENII $\backslash$ ix-w. in

GENI I Default Parameter Values (28-Mar-90 RAP)

Radionucl ide Library - Times $<100$ years (23-July-93 PDR)

External Dose Factors for GENII in person Sv/yr per Bq/n (8-May-90 $\mathrm{R}$

Worst-Case Solubilities, Yearly Dose Increments (23-Ju1-93 PDR) 
WHC-SD-WM-CN-024

Rev. 0

Page 18 of 36

\begin{tabular}{|c|c|c|c|}
\hline $\begin{array}{l}\text { Release } \\
\text { Radio- } \\
\text { nuclide }\end{array}$ & $\stackrel{\operatorname{Air}}{\mathrm{Ci} / \mathrm{yr}}$ & $\begin{array}{l}\text { Surface } \\
\text { Water } \\
\mathrm{Ci} / \mathrm{yr}\end{array}$ & $\begin{array}{r}\text { Buried } \\
\text { Source } \\
\mathrm{C} i / \mathrm{m} 3\end{array}$ \\
\hline $\begin{array}{l}\text { CO60 } \\
\text { SR90 } \\
Y \quad 90 \\
C S 137 \\
\text { PA233 } \\
\text { U } 234 \\
U 235 \\
\text { U } 236 \\
U 238 \\
\text { NP237 } \\
\text { PU238 } \\
\text { PU239 } \\
\text { PU240 } \\
\text { PU241 } \\
\text { PU242 } \\
\text { AM241 }\end{array}$ & $\begin{array}{l}1.3 \mathrm{E}-04 \\
3.4 \mathrm{E}-01 \\
3.4 \mathrm{E}-01 \\
2.1 \mathrm{E}+00 \\
2.3 \mathrm{E}-06 \\
1.5 \mathrm{E}-05 \\
6.7 \mathrm{E}-07 \\
3.4 \mathrm{E}-07 \\
1.5 \mathrm{E}-05 \\
2.3 \mathrm{E}-06 \\
4.3 \mathrm{E}-03 \\
4.9 \mathrm{E}-02 \\
2.2 \mathrm{E}-02 \\
4.5 \mathrm{E}-01 \\
6.4 \mathrm{E}-06 \\
2.4 \mathrm{E}-01\end{array}$ & $\begin{array}{l}0.0 \mathrm{E}+00 \\
0.0 \mathrm{E}+00 \\
0.0 \mathrm{E}+00 \\
0.0 \mathrm{E}+00 \\
0.0 \mathrm{E}+00 \\
0.0 \mathrm{E}+00 \\
0.0 \mathrm{E}+00 \\
0.0 \mathrm{E}+00 \\
0.0 \mathrm{E}+00 \\
0.0 \mathrm{E}+00 \\
0.0 \mathrm{E}+00 \\
0.0 \mathrm{E}+00 \\
0.0 \mathrm{E}+00 \\
0.0 \mathrm{E}+00 \\
0.0 \mathrm{E}+00 \\
0.0 \mathrm{E}+00\end{array}$ & $\begin{array}{l}0.0 \mathrm{E}+00 \\
0.0 \mathrm{E}+00 \\
0.0 \mathrm{E}+00 \\
0.0 \mathrm{E}+00 \\
0.0 \mathrm{E}+00 \\
0.0 \mathrm{E}+00 \\
0.0 \mathrm{E}+00 \\
0.0 \mathrm{E}+00 \\
0.0 \mathrm{E}+00 \\
0.0 \mathrm{E}+00 \\
0.0 \mathrm{E}+00 \\
0.0 \mathrm{E}+00 \\
0.0 \mathrm{E}+00 \\
0.0 \mathrm{E}+00 \\
0.0 \mathrm{E}+00 \\
0.0 \mathrm{E}+00\end{array}$ \\
\hline
\end{tabular}

$=x=======$ AIR TRANSPORT

$1.0 E+00$ Input $E / Q$ value $(\mathrm{s} / \mathrm{m} 3)$

$===\pi=====$ EXTERNAL EXPOSURE

1.0E+00 Fraction of time spent in cloud

INHALATION

Resuspension not considered

Input prepared by:

Date:

Input checked by:

Date: 
WHC-SD-WM-CN-024

Rev. 0

Page 19 of 36

\section{GENII Dose Calculation Program}

(Version 1.485 3-Dec-90)

Case title: Ion Exchange Column $L 3$ - Worker

Executed on: $03 / 22 / 96$ at $11: 25: 51$

Page C. 1

Acute release

Uptake/exposure period:

Dose commitment period:

Dose units:

1.0

50.0

Rem

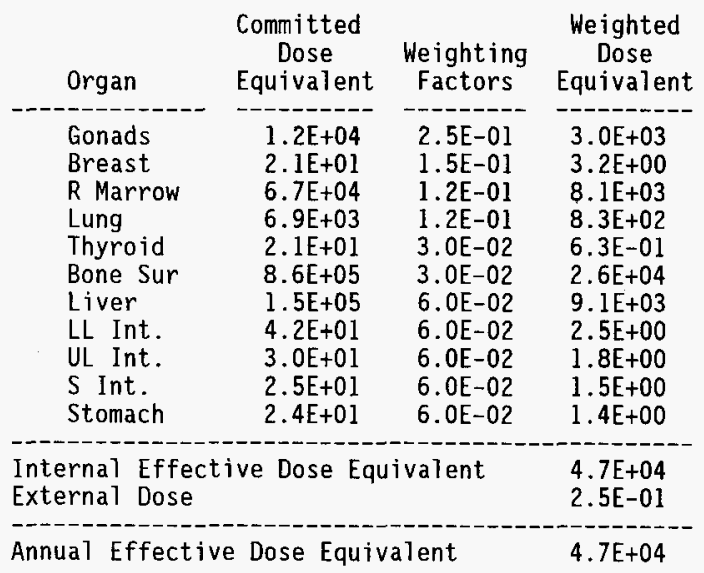

Controlling Organ:

Controlling Pathway:

Controlling Radionuclide:

Bone Sur

Inh

AM241

Total Inhalation EDE:

4. $7 E+04$

Total Ingestion EDE:

$0.0 \mathrm{E}+00$ 
WHC-SD-WM-CN-024

Rev. 0

Page 20 of 36

\section{GENI I Dose Calculation Program}

(Version 1.485 3-Dec-90)

Case title: Ion Exchange Column L3 - Worker

Executed on: $03 / 22 / 96$ at $11: 25: 51$

Page C. 2

Acute release

Uptake/exposure period:

Dose commitment period:

Dose units:
1.0

50.0

Dose Commitment Year

$1 \quad 3 \quad 3 \quad$.

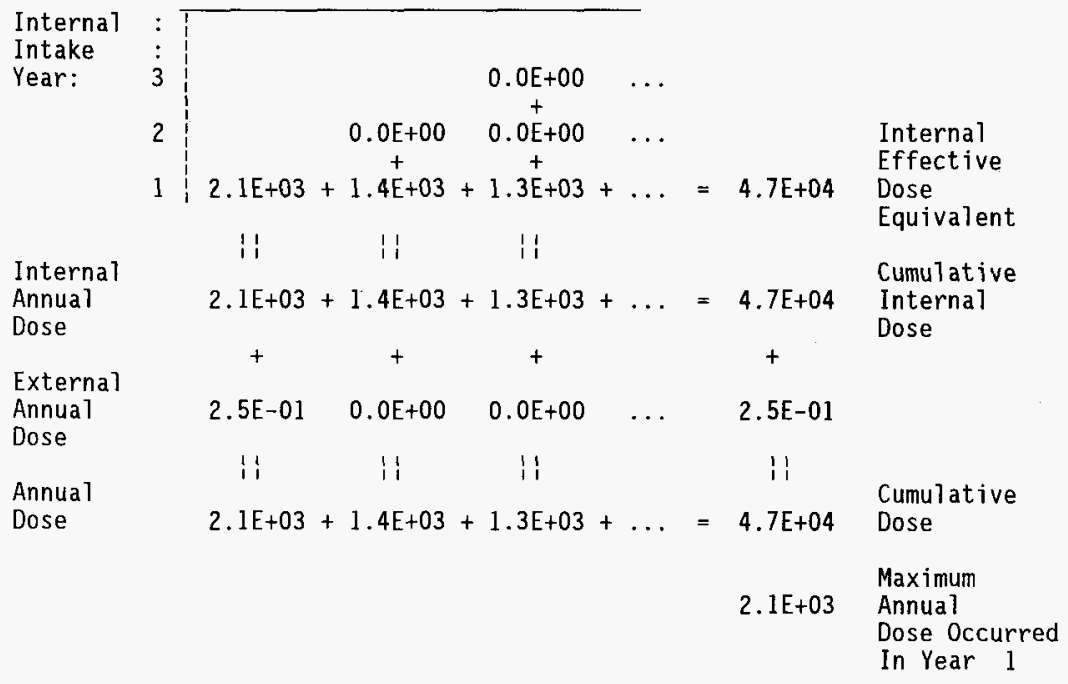


WHC-SD-WM-CN-024

Rev. 0

Page 21 of 36

\section{GENII Dose Calculation Program}

(Version 1.485 3-Dec-90)

Case title: Ion Exchange Column L3 - Worker

Executed on: $03 / 22 / 96$ at $11: 25: 51$

Page C. 3

Acute release

Uptake/exposure period:

Dose commitment period:

Dose units:

\section{Inhalation Ingestion}

Effective Effective

Radio-

nuclide

-------.-

CO 60

SR 90

Y 90

CS 137

PU 240

U 236

PU 238

PU 242

U 234

U 238

TH 234

PA 234

PU 241

U 237

AM 241

NP 237

PA 233

PU 239

U 235

TH 231
Dose

Equivalent

8.7E-03

2. $2 \mathrm{E}+01$

9.6E-01

2. $1 \mathrm{E}+01$

3. $2 \mathrm{E}+03$

1.4E-02

5. $5 \mathrm{E}+02$

8. $5 \mathrm{E}-01$

6.5E-01

5. $8 \mathrm{E}-01$

$0.0 \mathrm{E}+00$

$0.0 E+00$

1. $2 \mathrm{E}+03$

$0.0 E+00$

$3.5 \mathrm{E}+04$

4. $8 \mathrm{E}-01$

7. $3 \mathrm{E}-06$

6. $9 \mathrm{E}+03$

2.7 E-02

$0.0 \mathrm{E}+00$
Dose

Equivalent

----------

$0.0 \mathrm{E}+00$

$0.0 \mathrm{E}+00$

$0.0 \mathrm{E}+00$

$0.0 \mathrm{E}+00$

$0.0 \mathrm{E}+00$

$0.0 \mathrm{E}+00$

$0.0 \mathrm{E}+00$

$0.0 \mathrm{E}+00$

$0.0 \mathrm{E}+00$

$0.0 \mathrm{E}+00$

$0.0 \mathrm{E}+00$

$0.0 \mathrm{E}+00$

$0.0 \mathrm{E}+00$

$0.0 \mathrm{E}+00$

$0.0 \mathrm{E}+00$

$0.0 \mathrm{E}+00$

$0.0 \mathrm{E}+00$

$0.0 \mathrm{E}+00$

$0.0 \mathrm{E}+00$

$0.0 \mathrm{E}+00$
External

Dose

$6.5 \mathrm{E}-05$

1. $4 \mathrm{E}-05$

5. $2 \mathrm{E}-04$

2. $4 \mathrm{E}-01$

$6.8 \mathrm{E}-07$

$1.1 \mathrm{E}-11$

1. $4 \mathrm{E}-07$

$0.0 \mathrm{E}+00$

6. $3 \mathrm{E}-10$

4. $3 \mathrm{E}-10$

$0.0 \mathrm{E}+00$

$0.0 \mathrm{E}+00$

3. $4 \mathrm{E}-1]$

$0.0 \mathrm{E}+00$

5. $3 \mathrm{E}-04$

$0.0 \mathrm{E}+00$

1. $1 \mathrm{E}-07$

1.1E-06

1. $3 \mathrm{E}-08$

$0.0 \mathrm{E}+00$
1.0

50.0
Internal Annual

Effective Effective

Dose

Equivalent Equivalent

8.7E-03 8.7E-03

2. $2 \mathrm{E}+01 \quad 2.2 \mathrm{E}+01$

9. $6 \mathrm{E}-01 \quad 9.6 \mathrm{E}-01$

2. $1 E+01 \quad 2.1 E+01$

3. $2 \mathrm{E}+03 \quad 3.2 \mathrm{E}+03$

1. $4 \mathrm{E}-02 \quad 1.4 \mathrm{E}-02$

$5.5 \mathrm{E}+02 \quad 5.5 \mathrm{E}+02$

8.5E-01 8.5E-01

6.5E-01 6.5E-01

$5.8 \mathrm{E}-01 \quad 5.8 \mathrm{E}-01$

$0.0 E+00 \quad 0.0 E+00$

$0.0 \mathrm{E}+00 \quad 0.0 \mathrm{E}+00$

1. $2 \mathrm{E}+03 \quad 1.2 \mathrm{E}+03$

$0.0 \mathrm{E}+00 \quad 0.0 \mathrm{E}+00$

$3.5 \mathrm{E}+04 \quad 3.5 \mathrm{E}+04$

4.8E-01 4.8E-01

7. $3 \mathrm{E}-06 \quad 7.4 \mathrm{E}-06$

$6.9 \mathrm{E}+03 \quad 6.9 \mathrm{E}+03$

$2.7 \mathrm{E}-02 \quad 2.7 \mathrm{E}-02$

$0.0 \mathrm{E}+00 \quad 0.0 \mathrm{E}+00$ 
WHC-SD-WM-CN-024

Rev. 0

Page 22 of 36

Run started at 15:51:02 03/27/96

ISO-PC Version 1.98

August 1994

originally ISOSHLD-II; RIBD was removed

Please send questions or comments to:

Paul D. Rittmann, PhD CHP 509-376-8715

Westinghouse Hanford Company H0-36

PO Box 1970 Richland, WA 99352

Title Line from Library File (ISO-PC.LIB):

Attenuation \& Buildup for 30 Groups; Photon \& Beta Production 7/6/94 PDR

Run Title: IX Column Transfer Box

$L-3$

Table of Source Activity:

Scale Factor $=1.000 \mathrm{E}+00$

\begin{tabular}{|c|c|c|}
\hline $\begin{array}{l}\text { Isotope } \\
\text { Name }\end{array}$ & $\begin{array}{l}\text { Initial } \\
\text { Values }\end{array}$ & $\begin{array}{l}\text { Final } \\
\text { Curies }\end{array}$ \\
\hline $\begin{array}{l}\text { CO- } 60 \\
\text { SR- } 90 \\
Y-90 \\
\text { CS- } 137 \\
\text { BA-137M } \\
P A-233 \\
U-234 \\
U-235 \\
U-238 \\
\text { NP-237 } \\
\text { PU-238 } \\
\text { PU-239 } \\
\text { PU-240 } \\
\text { PU-241 } \\
\text { PU-242 } \\
\text { AM-241 }\end{array}$ & $\begin{array}{l}1.01 \mathrm{E}-03 \\
4.17 \mathrm{E}-01 \\
4.17 \mathrm{E}-01 \\
2.58 \mathrm{E}+00 \\
2.45 \mathrm{E}+00 \\
2.32 \mathrm{E}-06 \\
1.51 \mathrm{E}-05 \\
6.69 \mathrm{E}-07 \\
1.55 \mathrm{E}-05 \\
2.32 \mathrm{E}-06 \\
4.25 \mathrm{E}-03 \\
4.91 \mathrm{E}-02 \\
2.22 \mathrm{E}-02 \\
4.53 \mathrm{E}-01 \\
6.37 \mathrm{E}-06 \\
2.40 \mathrm{E}-01\end{array}$ & $\begin{array}{l}1.010 \mathrm{E}-03 \\
4.170 \mathrm{E}-01 \\
4.170 \mathrm{E}-01 \\
2.580 \mathrm{E}+00 \\
2.450 \mathrm{E}+00 \\
2.320 \mathrm{E}-06 \\
1.510 \mathrm{E}-05 \\
6.690 \mathrm{E}-07 \\
1.550 \mathrm{E}-05 \\
2.320 \mathrm{E}-06 \\
4.250 \mathrm{E}-03 \\
4.910 \mathrm{E}-02 \\
2.220 \mathrm{E}-02 \\
4.530 \mathrm{E}-01 \\
6.370 \mathrm{E}-06 \\
2.400 \mathrm{E}-01\end{array}$ \\
\hline
\end{tabular}




\section{$L-3$}

Shield Composition, $\mathrm{g} / \mathrm{cc}$

Shield 1 Shield 2

\begin{tabular}{lll} 
AIR & $0.000 \mathrm{E}+00$ & $1.290 \mathrm{E}-03$ \\
HAN SOIL & $1.000 \mathrm{E}+00$ & $0.000 \mathrm{E}+00$ \\
\hline Totals: & $1.000 \mathrm{E}+00$ & $1.290 \mathrm{E}-03$ \\
\hline
\end{tabular}

$\mathrm{E}, \mathrm{MeV}$ Linear Attenuation, per $\mathrm{cm}$ (last region is air)

$\begin{array}{lll}0.015 & 1.220 \mathrm{E}+01 & 2.046 \mathrm{E}-03 \\ 0.025 & 2.835 \mathrm{E}+00 & 6.138 \mathrm{E}-04 \\ 0.035 & 1.135 \mathrm{E}+00 & 3.664 \mathrm{E}-04 \\ 0.045 & 6.158 \mathrm{E}-01 & 2.837 \mathrm{E}-04 \\ 0.055 & 4.058 \mathrm{E}-01 & 2.491 \mathrm{E}-04 \\ 0.065 & 3.045 \mathrm{E}-01 & 2.314 \mathrm{E}-04 \\ 0.075 & 2.488 \mathrm{E}-01 & 2.184 \mathrm{E}-04 \\ 0.085 & 2.151 \mathrm{E}-01 & 2.090 \mathrm{E}-04 \\ 0.095 & 1.932 \mathrm{E}-01 & 2.019 \mathrm{E}-04 \\ 0.150 & 1.430 \mathrm{E}-01 & 1.758 \mathrm{E}-04 \\ 0.250 & 1.147 \mathrm{E}-01 & 1.477 \mathrm{E}-04 \\ 0.350 & 1.002 \mathrm{E}-01 & 1.298 \mathrm{E}-04 \\ 0.475 & 8.797 \mathrm{E}-02 & 1.146 \mathrm{E}-04 \\ 0.650 & 7.669 \mathrm{E}-02 & 1.001 \mathrm{E}-04 \\ 0.825 & 6.869 \mathrm{E}-02 & 8.984 \mathrm{E}-05 \\ 1.000 & 6.260 \mathrm{E}-02 & 8.194 \mathrm{E}-05 \\ 1.225 & 5.661 \mathrm{E}-02 & 7.398 \mathrm{E}-05 \\ 1.475 & 5.154 \mathrm{E}-02 & 6.718 \mathrm{E}-05 \\ 1.700 & 4.793 \mathrm{E}-02 & 6.249 \mathrm{E}-05 \\ 1.900 & 4.527 \mathrm{E}-02 & 5.889 \mathrm{E}-05 \\ 2.100 & 4.301 \mathrm{E}-02 & 5.564 \mathrm{E}-05 \\ 2.300 & 4.108 \mathrm{E}-02 & 5.263 \mathrm{E}-05 \\ 2.500 & 3.940 \mathrm{E}-02 & 5.003 \mathrm{E}-05 \\ 2.700 & 3.795 \mathrm{E}-02 & 4.778 \mathrm{E}-05 \\ 3.000 & 3.610 \mathrm{E}-02 & 4.511 \mathrm{E}-05 \\ 3.600 & 3.327 \mathrm{E}-02 & 4.158 \mathrm{E}-05 \\ 4.500 & 3.026 \mathrm{E}-02 & 3.746 \mathrm{E}-05 \\ 5.500 & 2.796 \mathrm{E}-02 & 3.381 \mathrm{E}-05 \\ 6.600 & 2.624 \mathrm{E}-02 & 3.111 \mathrm{E}-05 \\ 8.600 & 2.436 \mathrm{E}-02 & 2.771 \mathrm{E}-05 \\ ----------------\end{array}$


WHC-SD-WM-CN-024

Rev. 0

Page 24 of 36

$L-3$

Photon Production Rate for Each Radionuclide:

ISOSHLD Energy Group Structure:

\begin{tabular}{|c|c|c|c|c|c|c|c|}
\hline $\begin{array}{l}\text { Group } \\
\text { Number }\end{array}$ & $\begin{array}{l}\text { Photon } \\
\text { Low }\end{array}$ & $\underset{\text { Mid }}{\text { Energy, }}$ & $\begin{array}{l}\text { Mev } \\
\text { High }\end{array}$ & $\begin{array}{l}\text { Group } \\
\text { Number }\end{array}$ & $\begin{array}{l}\text { Photon } \\
\text { Low }\end{array}$ & $\begin{array}{l}\text { Energy, } \\
\text { Mid }\end{array}$ & $\begin{array}{l}\text { Mev } \\
\text { High }\end{array}$ \\
\hline 1 & 0.010 & 0.015 & 0,020 & 16 & 0.900 & 1000 & 1.10 \\
\hline 2 & 0.020 & 0.025 & 0.030 & 17 & 1.100 & & \\
\hline 3 & 0.030 & 0.035 & 0.040 & 18 & 1.350 & 1.475 & 1.6 \\
\hline 4 & 0.040 & 0.045 & 0.050 & 19 & 1.600 & 1.700 & \\
\hline 5 & 0.050 & 0.055 & 0.060 & 20 & 1.800 & 1.900 & 2.0 \\
\hline 6 & 0.060 & 0.065 & 0.070 & 21 & 2.000 & 2.100 & \\
\hline 7 & 0.070 & 0.075 & 0.080 & 22 & 2.200 & 2.300 & 2.4 \\
\hline 8 & 0.080 & 0.085 & 0.090 & 23 & 2.400 & 2.500 & 2.6 \\
\hline 9 & 0.090 & 0.095 & 0.100 & 24 & 2.600 & 2.700 & 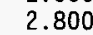 \\
\hline 10 & 0.100 & 0.150 & 0.200 & 25 & 2.800 & 3.000 & 3.2 \\
\hline 11 & 0.200 & 0.250 & 0.300 & 26 & 3.200 & 3.6 & \\
\hline 12 & 0.300 & 0.350 & 0.400 & 27 & 4.000 & 4.500 & \\
\hline 13 & 0.400 & 0.475 & 0.550 & 28 & 5.000 & 5.500 & 6 \\
\hline 14 & 0.550 & 0.650 & 0.750 & 29 & 6.000 & 6.600 & bo \\
\hline 15 & 0.750 & 0.825 & 0.900 & 30 & 7.200 & 8.600 & 10.000 \\
\hline
\end{tabular}

\begin{tabular}{|c|c|c|c|c|c|c|c|}
\hline & & & & & & tencos & \\
\hline $\begin{array}{l}\text { Group } \\
\text { No. }\end{array}$ & $\begin{array}{l}\text { Gamma } \\
\text { X-ray }\end{array}$ & Brems & $\begin{array}{c}\text { Total } \\
\text { photon/sec }\end{array}$ & $\begin{array}{l}\text { Group } \\
\text { No. }\end{array}$ & $\begin{array}{l}\text { Gamma } \\
\text { X-ray }\end{array}$ & Brems & $\begin{array}{c}\text { Total } \\
\text { photon/sec }\end{array}$ \\
\hline $\begin{array}{rl}1 & 0 \\
2 & 0 \\
3 & 0 \\
4 & 0 \\
5 & 0 \\
6 & 0 \\
7 & 0 \\
8 & 0 \\
9 & 0 \\
10 & 0 \\
11 & 0 \\
12 & 0 \\
15 & 3\end{array}$ & & $\begin{array}{l}3.16 \\
1.27 \\
8.21 \\
5.72 \\
3.95 \\
2.82 \\
2.05 \\
5.34 \\
1.42 \\
5.63 \\
0.00\end{array}$ & & $\begin{array}{ll}16 & 0 \\
17 & 7 \\
18 & 0 \\
19 & 0 \\
20 & 0 \\
21 & 0 \\
22 & 0 \\
23 & 0 \\
24 & 0 \\
25 & 0 \\
26 & 0 \\
27 & 0 \\
30 & 0\end{array}$ & & $\begin{array}{l}.000 E+00 \\
.000 E+00 \\
.000 E+00 \\
.000 E+00 \\
.000 E+00 \\
.000 E+00 \\
.000 E+00 \\
.000 E+00 \\
.000 E+00 \\
.000 E+00 \\
.000 E+00 \\
.000 E+00 \\
.000 E+00\end{array}$ & $\begin{array}{l}0.000 \mathrm{E}+00 \\
7.474 \mathrm{E}+07 \\
0.000 \mathrm{E}+00 \\
0.000 \mathrm{E}+00 \\
0.000 \mathrm{E}+00 \\
0.000 \mathrm{E}+00 \\
0.000 \mathrm{E}+00 \\
0.000 \mathrm{E}+00 \\
0.000 \mathrm{E}+00 \\
0.000 \mathrm{E}+00 \\
0.000 \mathrm{E}+00 \\
0.000 \mathrm{E}+00 \\
0.000 \mathrm{E}+00\end{array}$ \\
\hline
\end{tabular}

Totals: $7.474 \mathrm{E}+07 \quad 2.180 \mathrm{E}+05 \quad 7.496 \mathrm{E}+07$ 
WHC-SD-WM-CN-024

Rev. 0

Page 25 of 36

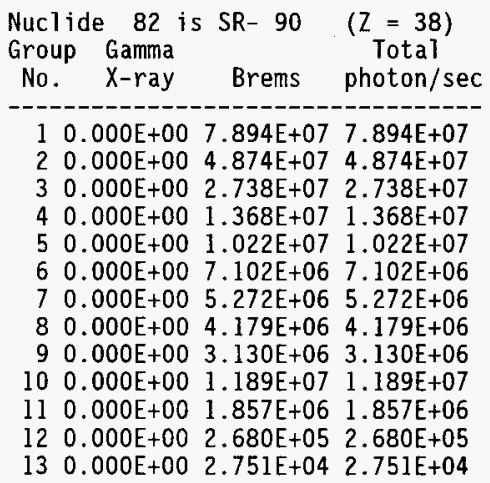

\begin{tabular}{cccc}
$\begin{array}{c}\text { Weight }(82) \\
\text { Group Gamma } \\
\text { No. }\end{array}$ X-ray & Brems & $\begin{array}{c}\text { Total } \\
\text { photon } / \mathrm{sec}\end{array}$ \\
\hdashline $170 \mathrm{E}-01 \mathrm{C}$ & $0.000 \mathrm{E}+00$ & $0.000 \mathrm{E}+00$ & $0.000 \mathrm{E}+00$ \\
17 & $0.000 \mathrm{E}+00$ & $0.000 \mathrm{E}+00$ & $0.000 \mathrm{E}+00$ \\
18 & $0.000 \mathrm{E}+00$ & $0.000 \mathrm{E}+00$ & $0.000 \mathrm{E}+00$ \\
19 & $0.000 \mathrm{E}+00$ & $0.000 \mathrm{E}+00$ & $0.000 \mathrm{E}+00$ \\
20 & $0.000 \mathrm{E}+00$ & $0.000 \mathrm{E}+00$ & $0.000 \mathrm{E}+00$ \\
21 & $0.000 \mathrm{E}+00$ & $0.000 \mathrm{E}+00$ & $0.000 \mathrm{E}+00$ \\
22 & $0.000 \mathrm{E}+00$ & $0.000 \mathrm{E}+00$ & $0.000 \mathrm{E}+00$ \\
23 & $0.000 \mathrm{E}+00$ & $0.000 \mathrm{E}+00$ & $0.000 \mathrm{E}+00$ \\
24 & $0.000 \mathrm{E}+00$ & $0.000 \mathrm{E}+00$ & $0.000 \mathrm{E}+00$ \\
25 & $0.000 \mathrm{E}+00$ & $0.000 \mathrm{E}+00$ & $0.000 \mathrm{E}+00$ \\
26 & $0.000 \mathrm{E}+00$ & $0.000 \mathrm{E}+00$ & $0.000 \mathrm{E}+00$ \\
27 & $0.000 \mathrm{E}+00$ & $0.000 \mathrm{E}+00$ & $0.000 \mathrm{E}+00$ \\
28 & $0.000 \mathrm{E}+00$ & $0.000 \mathrm{E}+00$ & $0.000 \mathrm{E}+00$
\end{tabular}

Totals: $0.000 \mathrm{E}+00 \quad 2.127 \mathrm{E}+08 \quad 2.127 \mathrm{E}+08$

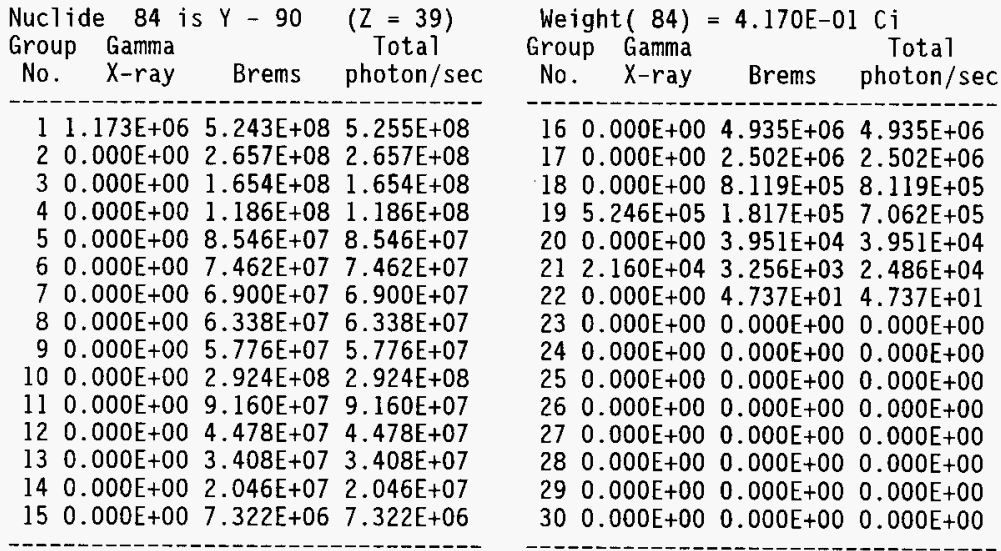




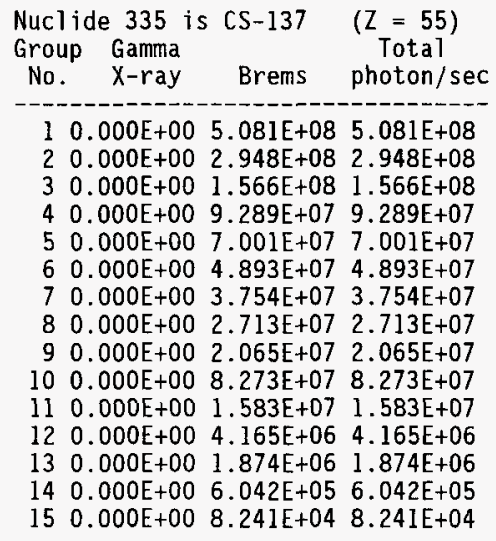

Weight $(335)=2.580 \mathrm{E}+00 \mathrm{Ci}$

Group Gamma Total

No. $x$-ray Brems photon/sec

$16 \quad 0.000 \mathrm{E}+00 \quad 1.203 \mathrm{E}+04 \quad 1.203 \mathrm{E}+04$

$170.000 E+008.789 E+018.789 E+01$

$180.000 \mathrm{E}+00 \quad 0.000 \mathrm{E}+00 \quad 0.000 \mathrm{E}+00$

$190.000 \mathrm{E}+00 \quad 0.000 \mathrm{E}+00 \quad 0.000 \mathrm{E}+00$

$20 \quad 0.000 \mathrm{E}+00 \quad 0.000 \mathrm{E}+00 \quad 0.000 \mathrm{E}+00$

$210.000 \mathrm{E}+00 \quad 0.000 \mathrm{E}+00 \quad 0.000 \mathrm{E}+00$

$22 \quad 0.000 \mathrm{E}+00 \quad 0.000 \mathrm{E}+00 \quad 0.000 \mathrm{E}+00$

$23 \quad 0.000 \mathrm{E}+00 \quad 0.000 \mathrm{E}+00 \quad 0.000 \mathrm{E}+00$

$24 \quad 0.000 \mathrm{E}+00 \quad 0.000 \mathrm{E}+00 \quad 0.000 \mathrm{E}+00$

$250.000 \mathrm{E}+00 \quad 0.000 \mathrm{E}+00 \quad 0.000 \mathrm{E}+00$

$26 \quad 0.000 E+00 \quad 0.000 E+00 \quad 0.000 E+00$

$270.000 \mathrm{E}+00 \quad 0.000 \mathrm{E}+00 \quad 0.000 \mathrm{E}+00$

$280.000 \mathrm{E}+00 \quad 0.000 \mathrm{E}+00 \quad 0.000 \mathrm{E}+00$

$290.000 E+00 \quad 0.000 E+00 \quad 0.000 E+00$

$30 \quad 0.000 \mathrm{E}+00 \quad 0.000 \mathrm{E}+00 \quad 0.000 \mathrm{E}+00$

Totals: $0.000 \mathrm{E}+00 \quad 1.362 \mathrm{E}+09 \quad 1.362 \mathrm{E}+09$

Nuclide 336 is BA-137M $(Z=56)$ Group Gamma

No. X-ray Brems photon/sec

$36.599 E+090.000 E+00 \quad 6.599 E+09$

$148.157 E+10 \quad 0.000 E+00 \quad 8.157 E+10$
Weight $(336)=2.450 \mathrm{E}+00 \mathrm{Ci}$ Group Gamma Total No. X-ray Brems photon/sec

$\begin{array}{llll}18 & 0.000 E+00 & 0.000 E+00 & 0.000 E+00\end{array}$ $29 \quad 0.000 \mathrm{E}+00 \quad 0.000 \mathrm{E}+00 \quad 0.000 \mathrm{E}+00$ 
WHC-SD-WM-CN-024

Rev. 0

Page 27 of 36

\begin{tabular}{|c|c|c|c|c|c|c|c|}
\hline $\begin{array}{l}\text { Nuc] id } \\
\text { Group } \\
\text { No. }\end{array}$ & $\begin{array}{l}\text { e } 490 \text { is } \\
\text { Gamma } \\
\text { X-ray }\end{array}$ & $\begin{array}{r}\text { PA-233 } \\
\text { Brems }\end{array}$ & $\begin{array}{l}(Z=91) \\
\text { Total } \\
\text { photon } / \mathrm{sec}\end{array}$ & $\begin{array}{l}\text { Weigh } \\
\text { Group } \\
\text { No. }\end{array}$ & $\begin{array}{r}\text { Gamma } \\
\text { X-ray }\end{array}$ & $\begin{array}{c}=2.320 \mathrm{E}-06 \\
\text { Brems }\end{array}$ & $\begin{array}{l}6 \mathrm{Ci} \\
\text { Total } \\
\text { photon/sec }\end{array}$ \\
\hline $\begin{array}{rl}1 & 4 . \\
2 & 0 . \\
3 & 0 . \\
4 & 0 . \\
5 & 0 . \\
6 & 0 . \\
7 & 1 . \\
8 & 1 . \\
9 & 2 . \\
10 & 7 . \\
11 & 2 . \\
12 & 4 . \\
13 & 1 . \\
14 & 0 .\end{array}$ & $\begin{array}{l}199 \mathrm{E}+04 \\
000 \mathrm{E}+00 \\
000 \mathrm{E}+00 \\
000 \mathrm{E}+00 \\
000 \mathrm{E}+00 \\
000 \mathrm{E}+00 \\
082 \mathrm{E}+03 \\
622 \mathrm{E}+03 \\
439 \mathrm{E}+04 \\
837 \mathrm{E}+03 \\
575 \mathrm{E}+02 \\
434 \mathrm{E}+04 \\
.391 \mathrm{E}+03 \\
.000 \mathrm{E}+00\end{array}$ & $\begin{array}{l}2.181 \mathrm{E}+02 \\
7.664 \mathrm{E}+01 \\
3.898 \mathrm{E}+01 \\
2.104 \mathrm{E}+01 \\
1.313 \mathrm{E}+01 \\
8.430 \mathrm{E}+00 \\
5.478 \mathrm{E}+00 \\
3.738 \mathrm{E}+00 \\
2.542 \mathrm{E}+00 \\
6.629 \mathrm{E}+00 \\
5.426 \mathrm{E}-01 \\
8.045 \mathrm{E}-02 \\
7.986 \mathrm{E}-03 \\
1.541 \mathrm{E}-06\end{array}$ & $\begin{array}{l}4.221 \mathrm{E}+04 \\
7.664 \mathrm{E}+01 \\
3.898 \mathrm{E}+01 \\
2.104 \mathrm{E}+01 \\
1.313 \mathrm{E}+01 \\
8.430 \mathrm{E}+00 \\
1.087 \mathrm{E}+03 \\
1.626 \mathrm{E}+03 \\
2.439 \mathrm{E}+04 \\
7.844 \mathrm{E}+03 \\
2.581 \mathrm{E}+02 \\
4.434 \mathrm{E}+04 \\
1.391 \mathrm{E}+03 \\
1.541 \mathrm{E}-06\end{array}$ & $\begin{array}{ll}16 & 0 . \\
17 & 0 . \\
18 & 0 . \\
19 & 0 . \\
20 & 0 . \\
21 & 0 . \\
22 & 0 . \\
23 & 0 . \\
24 & 0 . \\
25 & 0 . \\
26 & 0 . \\
27 & 0 . \\
28 & 0 . \\
29 & 0 .\end{array}$ & $\begin{array}{l}.000 \mathrm{E}+00 \\
.000 \mathrm{E}+00 \\
.000 \mathrm{E}+00 \\
.000 \mathrm{E}+00 \\
.000 \mathrm{E}+00 \\
.000 \mathrm{E}+00 \\
.000 \mathrm{E}+00 \\
.000 \mathrm{E}+00 \\
.000 \mathrm{E}+00 \\
.000 \mathrm{E}+00 \\
.000 \mathrm{E}+00 \\
.000 \mathrm{E}+00 \\
.000 \mathrm{E}+00 \\
.000 \mathrm{E}+00\end{array}$ & $\begin{array}{l}0.000 E+00 \\
0.000 E+00 \\
0.000 E+00 \\
0.000 E+00 \\
0.000 E+00 \\
0.000 E+00 \\
0.000 E+00 \\
0.000 E+00 \\
0.000 E+00 \\
0.000 E+00 \\
0.000 E+00 \\
0.000 E+00 \\
0.000 E+00 \\
0.000 E+00\end{array}$ & $\begin{array}{l}0.000 E+00 \\
0.000 E+00 \\
0.000 E+00 \\
0.000 E+00 \\
0.000 E+00 \\
0.000 E+00 \\
0.000 E+00 \\
0.000 E+00 \\
0.000 E+00 \\
0.000 E+00 \\
0.000 E+00 \\
0.000 E+00 \\
0.000 E+00 \\
0.000 E+00\end{array}$ \\
\hline
\end{tabular}

Totals: $1.229 \mathrm{E}+05 \quad 3.954 \mathrm{E}+02 \quad 1.233 \mathrm{E}+05$

\begin{tabular}{|c|c|c|c|c|c|c|c|}
\hline Nucli & le 520 is & s U -234 & $(Z=92)$ & Weigh & $n t(520)=$ & $=1.510 \mathrm{E}-05$ & $5 \mathrm{Ci}$ \\
\hline $\begin{array}{l}\text { Group } \\
\text { No. }\end{array}$ & $\begin{array}{l}\text { Gamma } \\
X \text {-ray }\end{array}$ & Brems & $\begin{array}{c}\text { Total } \\
\text { photon/sec }\end{array}$ & $\begin{array}{l}\text { Group } \\
\text { No. }\end{array}$ & $\begin{array}{l}\text { Gamma } \\
X \text {-ray }\end{array}$ & Brems & $\begin{array}{c}\text { Total } \\
\text { photon/sec }\end{array}$ \\
\hline $\begin{array}{rl}1 & 5 \\
2 & 0 \\
3 & 0 \\
4 & 0 \\
5 & 6 \\
6 & 0 \\
7 & 0 \\
8 & 0 \\
9 & 0 \\
10 & 2 \\
13 & 2 \\
14 & 6\end{array}$ & $\begin{array}{l}866 \mathrm{E}+04 \\
000 \mathrm{E}+00 \\
000 \mathrm{E}+00 \\
000 \mathrm{E}+00 \\
649 \mathrm{E}+02 \\
000 \mathrm{E}+00 \\
000 \mathrm{E}+00 \\
000 \mathrm{E}+00 \\
000 \mathrm{E}+00 \\
235 \mathrm{E}+02 \\
235 \mathrm{E}+01 \\
.04 \mathrm{E}-02\end{array}$ & $\begin{array}{l}4.933 \mathrm{E}+00 \\
4.609 \mathrm{E}-01 \\
5.537 \mathrm{E}-02 \\
1.066 \mathrm{E}-02 \\
3.376 \mathrm{E}-03 \\
1.013 \mathrm{E}-03 \\
2.355 \mathrm{E}-04 \\
3.335 \mathrm{E}-05 \\
1.538 \mathrm{E}-06 \\
5.702 \mathrm{E}-11 \\
0.000 \mathrm{E}+00 \\
0.000 \mathrm{E}+00\end{array}$ & $\begin{array}{l}5.867 \mathrm{E}+04 \\
4.609 \mathrm{E}-01 \\
5.537 \mathrm{E}-02 \\
1.066 \mathrm{E}-02 \\
6.649 \mathrm{E}+02 \\
1.013 \mathrm{E}-03 \\
2.355 \mathrm{E}-04 \\
3.335 \mathrm{E}-05 \\
1.538 \mathrm{E}-06 \\
2.235 \mathrm{E}+02 \\
2.235 \mathrm{E}+01 \\
6.704 \mathrm{E}-02\end{array}$ & $\begin{array}{ll}16 & 0 . \\
17 & 0 . \\
18 & 0 . \\
19 & 0 . \\
20 & 0 . \\
21 & 0 . \\
22 & 0 . \\
23 & 0 . \\
24 & 0 . \\
25 & 0 . \\
28 & 0 . \\
29 & 0 .\end{array}$ & $\begin{array}{l}.000 \mathrm{E}+00 \\
.000 \mathrm{E}+00 \\
.000 \mathrm{E}+00 \\
.000 \mathrm{E}+00 \\
.000 \mathrm{E}+00 \\
.000 \mathrm{E}+00 \\
.000 \mathrm{E}+00 \\
.000 \mathrm{E}+00 \\
.000 \mathrm{E}+00 \\
.000 \mathrm{E}+00 \\
.000 \mathrm{E}+00 \\
.000 \mathrm{E}+00\end{array}$ & $\begin{array}{l}0.000 \mathrm{E}+00 \\
0.000 \mathrm{E}+00 \\
0.000 \mathrm{E}+00 \\
0.000 \mathrm{E}+00 \\
0.000 \mathrm{E}+00 \\
0.000 \mathrm{E}+00 \\
0.000 \mathrm{E}+00 \\
0.000 \mathrm{E}+00 \\
0.000 \mathrm{E}+00 \\
0.000 \mathrm{E}+00 \\
0.000 \mathrm{E}+00 \\
0.000 \mathrm{E}+00\end{array}$ & $\begin{array}{l}0.000 \mathrm{E}+00 \\
0.000 \mathrm{E}+00 \\
0.000 \mathrm{E}+00 \\
0.000 \mathrm{E}+00 \\
0.000 \mathrm{E}+00 \\
0.000 \mathrm{E}+00 \\
0.000 \mathrm{E}+00 \\
0.000 \mathrm{E}+00 \\
0.000 \mathrm{E}+00 \\
0.000 \mathrm{E}+00 \\
0.000 \mathrm{E}+00 \\
0.000 \mathrm{E}+00\end{array}$ \\
\hline
\end{tabular}

Totals: $5.957 \mathrm{E}+04 \quad 5.465 \mathrm{E}+00 \quad 5.958 \mathrm{E}+04$ 
WHC-SD-WM-CN-024

Rev. 0

Page 28 of 36

\begin{tabular}{|c|c|c|c|c|c|c|}
\hline $\begin{array}{l}\text { Nucl ide } \\
\text { Group } \\
\text { No. }\end{array}$ & $\begin{array}{l}476 \text { is } \\
\text { Gamma } \\
\text { X-ray }\end{array}$ & $\begin{array}{r}\text { S }-235 \\
\text { Brems }\end{array}$ & $\begin{array}{c}(Z=92) \\
\text { Total } \\
\text { photon } / \mathrm{sec}\end{array}$ & $\begin{array}{l}\text { Weight }(476) \\
\text { Group Gamma } \\
\text { No. X-ray }\end{array}$ & $\begin{array}{c}=6.690 \mathrm{E}-07 \\
\text { Brems }\end{array}$ & $\begin{array}{l}7 \mathrm{Ci} \\
\text { Total } \\
\text { photon/sec }\end{array}$ \\
\hline $\begin{array}{rl}1 & 7.5 \\
2 & 0.0 \\
3 & 0.0 \\
4 & 0.0 \\
5 & 0.0 \\
6 & 0.0 \\
7 & 2.7 \\
8 & 0.5 \\
9 & 1.7 \\
10 & 1.8 \\
11 & 1.5 \\
12 & 3.2 \\
13 & 2.9 \\
14 & 9.5 \\
15 & 1.5\end{array}$ & $\begin{array}{l}651 E+03 \\
000 E+00 \\
000 E+00 \\
000 E+00 \\
000 E+00 \\
000 E+00 \\
723 E+01 \\
000 E+00 \\
780 E+03 \\
335 E+04 \\
500 E+03 \\
218 E+01 \\
970 E+00 \\
901 E-02 \\
485 E-01\end{array}$ & $\begin{array}{l}1.340 \mathrm{E}+00 \\
3.397 \mathrm{E}-01 \\
1.251 \mathrm{E}-01 \\
5.405 \mathrm{E}-02 \\
2.595 \mathrm{E}-02 \\
1.322 \mathrm{E}-02 \\
6.961 \mathrm{E}-03 \\
3.653 \mathrm{E}-03 \\
1.899 \mathrm{E}-03 \\
3.356 \mathrm{E}-03 \\
0.000 \mathrm{E}+00 \\
0.000 \mathrm{E}+00 \\
0.000 \mathrm{E}+00 \\
0.000 \mathrm{E}+00 \\
0.000 \mathrm{E}+00\end{array}$ & $\begin{array}{l}7.652 \mathrm{E}+03 \\
3.397 \mathrm{E}-01 \\
1.251 \mathrm{E}-01 \\
5.405 \mathrm{E}-02 \\
2.595 \mathrm{E}-02 \\
1.322 \mathrm{E}-02 \\
2.724 \mathrm{E}+01 \\
3.653 \mathrm{E}-03 \\
1.780 \mathrm{E}+03 \\
1.835 \mathrm{E}+04 \\
1.500 \mathrm{E}+03 \\
3.218 \mathrm{E}+01 \\
2.970 \mathrm{E}+00 \\
9.901 \mathrm{E}-02 \\
1.485 \mathrm{E}-01\end{array}$ & $\begin{array}{ll}16 & 0.000 \mathrm{E}+00 \\
17 & 0.000 \mathrm{E}+00 \\
18 & 0.000 \mathrm{E}+00 \\
19 & 0.000 \mathrm{E}+00 \\
20 & 0.000 \mathrm{E}+00 \\
21 & 0.000 \mathrm{E}+00 \\
22 & 0.000 \mathrm{E}+00 \\
23 & 0.000 \mathrm{E}+00 \\
24 & 0.000 \mathrm{E}+00 \\
25 & 0.000 \mathrm{E}+00 \\
26 & 0.000 \mathrm{E}+00 \\
27 & 0.000 \mathrm{E}+00 \\
28 & 0.000 \mathrm{E}+00 \\
29 & 0.000 \mathrm{E}+00 \\
30 & 0.000 \mathrm{E}+00\end{array}$ & $\begin{array}{l}0.000 E+00 \\
0.000 E+00 \\
0.000 E+00 \\
0.000 E+00 \\
0.000 E+00 \\
0.000 E+00 \\
0.000 E+00 \\
0.000 E+00 \\
0.000 E+00 \\
0.000 E+00 \\
0.000 E+00 \\
0.000 E+00 \\
0.000 E+00 \\
0.000 E+00 \\
0.000 E+00\end{array}$ & $\begin{array}{l}0.000 \mathrm{E}+00 \\
0.000 \mathrm{E}+00 \\
0.000 \mathrm{E}+00 \\
0.000 \mathrm{E}+00 \\
0.000 \mathrm{E}+00 \\
0.000 \mathrm{E}+00 \\
0.000 \mathrm{E}+00 \\
0.000 \mathrm{E}+00 \\
0.000 \mathrm{E}+00 \\
0.000 \mathrm{E}+00 \\
0.000 \mathrm{E}+00 \\
0.000 \mathrm{E}+00 \\
0.000 \mathrm{E}+00 \\
0.000 \mathrm{E}+00 \\
0.000 \mathrm{E}+00\end{array}$ \\
\hline \multicolumn{7}{|c|}{ Totals: $2.934 \mathrm{E}+04 \quad 1.914 \mathrm{E}+00 \quad 2.934 \mathrm{E}+04$} \\
\hline $\begin{array}{l}\text { Nucl ide } \\
\text { Group } \\
\text { No. }\end{array}$ & $\begin{array}{l}526 \text { is } \\
\text { Gamma } \\
X-r a y\end{array}$ & $\begin{array}{l}\text { U }-238 \\
\text { Brems }\end{array}$ & $\begin{array}{c}(Z=92) \\
\text { Total } \\
\text { photon } / \mathrm{sec}\end{array}$ & $\begin{array}{c}\text { Weight (526) } \\
\text { Group Gamma } \\
\text { No. X-ray }\end{array}$ & $\begin{array}{c}=1.550 \mathrm{E}-05 \\
\text { Brems }\end{array}$ & $\begin{array}{l}\mathrm{Ci} \\
\text { Total } \\
\text { photon/sec }\end{array}$ \\
\hline $\begin{array}{ll}1 & 5.0 \\
2 & 0.0 \\
3 & 0.0 \\
4 & 0.0 \\
5 & 0.0 \\
6 & 5.7 \\
7 & 0.0 \\
8 & 0.0\end{array}$ & $\begin{array}{l}54 E+04 \\
00 E+00 \\
00 E+00 \\
00 E+00 \\
00 E+00 \\
735 E+02 \\
00 E+00 \\
00 E+00\end{array}$ & $\begin{array}{l}2.929 \mathrm{E}+00 \\
2.422 \mathrm{E}-01 \\
2.754 \mathrm{E}-02 \\
6.281 \mathrm{E}-03 \\
1.722 \mathrm{E}-03 \\
3.554 \mathrm{E}-04 \\
4.507 \mathrm{E}-05 \\
2.182 \mathrm{E}-06\end{array}$ & $\begin{array}{l}5.064 \mathrm{E}+04 \\
2.422 \mathrm{E}-01 \\
2.754 \mathrm{E}-02 \\
6.281 \mathrm{E}-03 \\
1.722 \mathrm{E}-03 \\
5.735 \mathrm{E}+02 \\
4.507 \mathrm{E}-05 \\
2.182 \mathrm{E}-06\end{array}$ & $\begin{array}{ll}16 & 0.000 \mathrm{E}+00 \\
17 & 0.000 \mathrm{E}+00 \\
18 & 0.000 \mathrm{E}+00 \\
19 & 0.000 \mathrm{E}+00 \\
20 & 0.000 \mathrm{E}+00 \\
21 & 0.000 \mathrm{E}+00 \\
22 & 0.000 \mathrm{E}+00 \\
23 & 0.000 \mathrm{E}+00\end{array}$ & $\begin{array}{l}0.000 E+00 \\
0.000 E+00 \\
0.000 E+00 \\
0.000 E+00 \\
0.000 E+00 \\
0.000 E+00 \\
0.000 E+00 \\
0.000 E+00\end{array}$ & $\begin{array}{l}0.000 \mathrm{E}+00 \\
0.000 \mathrm{E}+00 \\
0.000 \mathrm{E}+00 \\
0.000 \mathrm{E}+00 \\
0.000 \mathrm{E}+00 \\
0.000 \mathrm{E}+00 \\
0.000 \mathrm{E}+00 \\
0.000 \mathrm{E}+00\end{array}$ \\
\hline
\end{tabular}

Totals: $5.121 \mathrm{E}+04 \quad 3.207 \mathrm{E}+00 \quad 5.122 \mathrm{E}+04$ 
WHC-SD-WM-CN-024

Rev. 0

Page 29 of 36

\begin{tabular}{|c|c|c|c|c|c|c|c|}
\hline $\begin{array}{l}\text { Nucli } \\
\text { Group } \\
\text { No. }\end{array}$ & $\begin{array}{l}\text { e } 502 \text { is } \\
\text { Gamma } \\
\text { X-ray }\end{array}$ & $\begin{array}{r}\text { S NP-237 } \\
\text { Brems }\end{array}$ & $\begin{array}{c}(Z=93) \\
\text { Total } \\
\text { photon } / \mathrm{sec}\end{array}$ & $\begin{array}{l}\text { Weigh } \\
\text { Group } \\
\text { No. }\end{array}$ & $\begin{array}{l}t(502)= \\
\text { Gamma } \\
\text { X-ray }\end{array}$ & $=2.320 \mathrm{E}-06$ & $\begin{array}{l}6 \mathrm{Ci} \\
\text { Total } \\
\text { photon/sec }\end{array}$ \\
\hline $\begin{array}{rr}1 & 5 \\
2 & 1 \\
3 & 0 \\
4 & 1 \\
5 & 3 \\
6 & 5 \\
7 & 0 \\
8 & \\
9 & \\
10 & 2 \\
11 & \end{array}$ & $\begin{array}{l}1 E+04 \\
1 E+04 \\
0 E+00 \\
2 E+02 \\
5 E+02 \\
0 E+00 \\
0 E+00 \\
5 E+04 \\
2 E+03\end{array}$ & $\begin{array}{l}6.13 \\
1.07 \\
2.51 \\
6.74 \\
2.03 \\
6.59 \\
2.20 \\
7.13 \\
1.75 \\
6.81 \\
0.00\end{array}$ & $\begin{array}{l}5.08 \\
1.20 \\
2.51 \\
1.20 \\
3.60 \\
6.59 \\
2.20 \\
1.09 \\
4.29\end{array}$ & $\begin{array}{ll}16 & 0 . \\
17 & 0 . \\
18 & 0 . \\
19 & 0 . \\
20 & 0 . \\
21 & 0 . \\
22 & 0 . \\
23 & 0 . \\
24 & 0 . \\
25 & 0 . \\
26 & 0 .\end{array}$ & & & $\begin{array}{l}0.000 E+00 \\
0.000 E+00 \\
0.000 E+00 \\
0.000 E+00 \\
0.000 E+00 \\
0.000 E+00 \\
0.000 E+00 \\
0.000 E+00 \\
0.000 E+00 \\
0.000 E+00 \\
0.000 E+00\end{array}$ \\
\hline
\end{tabular}

Totals: $8.151 \mathrm{E}+04 \quad 7.562 \mathrm{E}+00 \quad 8.152 \mathrm{E}+04$

\begin{tabular}{|c|c|c|c|c|c|c|c|}
\hline $\begin{array}{l}\text { Nucl ide } \\
\text { Group } \\
\text { No. }\end{array}$ & $\begin{array}{l}\text { e } 492 \text { is } \\
\text { Gamma } \\
\text { X-ray }\end{array}$ & & $\begin{array}{c}(Z=94) \\
\text { Total } \\
\text { photon } / \mathrm{sec}\end{array}$ & $\begin{array}{l}\text { Weigh } \\
\text { Group } \\
\text { No. }\end{array}$ & $\begin{array}{l}t(492) \\
\text { Gamma } \\
\text { X-ray }\end{array}$ & $=4.250 \mathrm{E}-0$ & $\begin{array}{l}3 \mathrm{Ci} \\
\text { Total } \\
\text { photon/sec }\end{array}$ \\
\hline $\begin{array}{rr}1 & 1 \\
4 & 6 \\
10 & 1\end{array}$ & & & $\begin{array}{l}1.8 \\
6.29 \\
15\end{array}$ & $\begin{array}{ll}16 & 0 \\
19 & 0 \\
25 & 0\end{array}$ & & & \\
\hline
\end{tabular}

Totals: $1.832 \mathrm{E}+07 \quad 0.000 \mathrm{E}+00 \quad 1.832 \mathrm{E}+07$

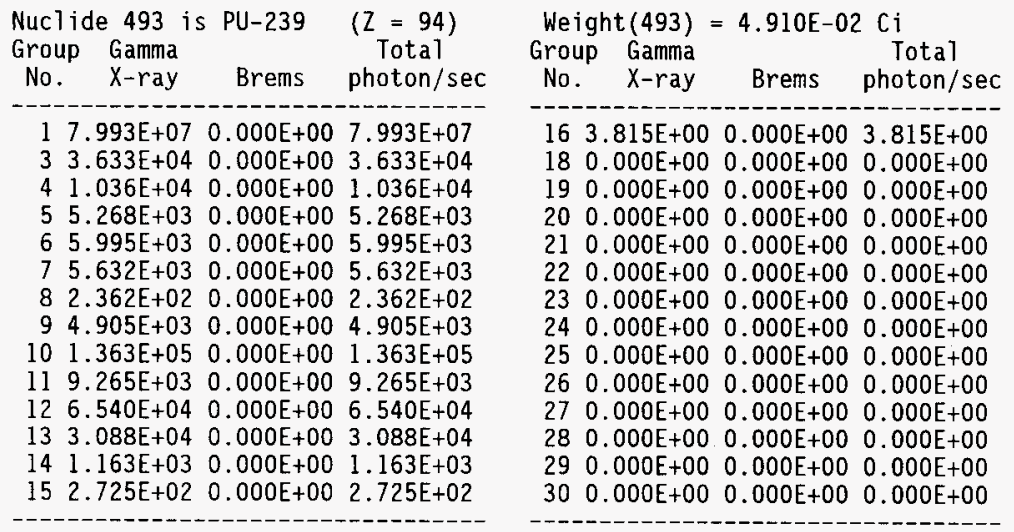


WHC-SD-WM-CN-024

Rev. 0

Page 30 of 36

\begin{tabular}{|c|c|c|c|c|c|c|}
\hline $\begin{array}{l}\text { Nucl ide } \\
\text { Group } \\
\text { No. }\end{array}$ & $\begin{array}{l}\text { e } 494 \text { is } \\
\text { Gamma } \\
\text { X-ray }\end{array}$ & Brems & $\begin{array}{l}(Z=94) \\
\text { Total } \\
\text { photon } / \mathrm{sec}\end{array}$ & $\begin{array}{c}\text { Weight (494) }= \\
\text { Group Gamma } \\
\text { No. X-ray }\end{array}$ & Brems & $\begin{array}{l}2 \mathrm{Cj} \\
\text { Total } \\
\text { photon/sec }\end{array}$ \\
\hline $\begin{array}{rl}4 & 3 . \\
10 & 6 . \\
14 & 1 .\end{array}$ & & VOU & & $\begin{array}{ll}19 & 0.000 \mathrm{E} \\
25 & 0.000 \mathrm{E} \\
29 & 0.000 \mathrm{E}\end{array}$ & & \\
\hline
\end{tabular}

Totals: $4.322 \mathrm{E}+05 \quad 0.000 \mathrm{E}+00 \quad 4.322 \mathrm{E}+05$

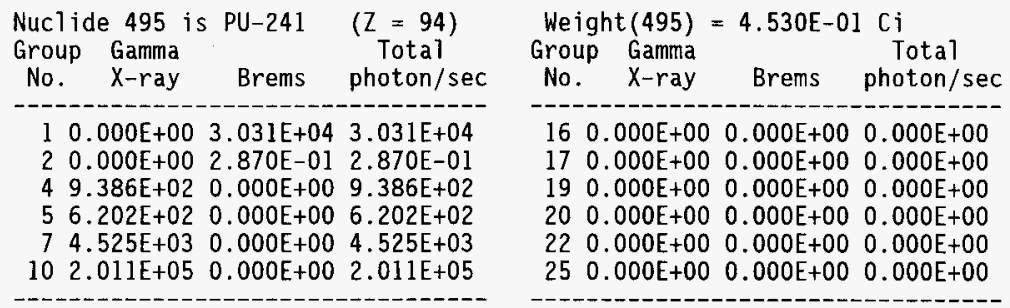

Totals: $2.072 \mathrm{E}+05 \quad 3.031 \mathrm{E}+04 \quad 2.375 \mathrm{E}+05$

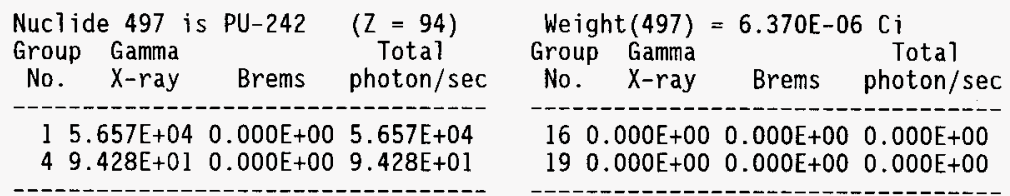




\section{WHC-SD-WM-CN-024 \\ Rev. 0 \\ Page 31 of 36}

\begin{tabular}{|c|c|c|c|c|c|c|c|}
\hline $\begin{array}{l}\text { Nucl id } \\
\text { Group } \\
\text { No. }\end{array}$ & $\begin{array}{l}\text { e } 496 \text { is } \\
\text { Gamma } \\
\text { X-ray }\end{array}$ & $\begin{array}{l}\text { AM-241 } \\
\text { Brems }\end{array}$ & $\begin{array}{c}(Z=95) \\
\text { Total } \\
\text { photon/sec }\end{array}$ & $\begin{array}{l}\text { Weigh } \\
\text { Group } \\
\text { No. }\end{array}$ & $\begin{array}{r}t(496) \\
\text { Gamma } \\
x-r a y\end{array}$ & $\begin{array}{c}=2.400 \mathrm{E}-0 \\
\text { Brems }\end{array}$ & $\begin{array}{c}\text { Ci } \\
\text { Total } \\
\text { photon/sec }\end{array}$ \\
\hline $\begin{array}{rl}1 & 3 . \\
2 & 2 . \\
3 & 9 . \\
4 & 7 . \\
5 & 3 . \\
6 & 1 . \\
7 & 0 . \\
8 & 0 . \\
9 & 1 . \\
10 & 2 . \\
11 & 7 . \\
12 & 1 . \\
13 & 6 . \\
14 & 6 . \\
15 & 1 .\end{array}$ & $\begin{array}{l}818 \mathrm{E}+09 \\
131 \mathrm{E}+08 \\
768 \mathrm{E}+06 \\
992 \mathrm{E}+06 \\
170 \mathrm{E}+09 \\
776 \mathrm{E}+05 \\
000 \mathrm{E}+00 \\
000 \mathrm{E}+00 \\
865 \mathrm{E}+06 \\
486 \mathrm{E}+06 \\
637 \mathrm{E}+04 \\
154 \mathrm{E}+05 \\
926 \mathrm{E}+03 \\
127 \mathrm{E}+04 \\
954 \mathrm{E}+03\end{array}$ & $\begin{array}{l}1.800 \mathrm{E}+05 \\
1.877 \mathrm{E}+04 \\
2.216 \mathrm{E}+03 \\
2.420 \mathrm{E}+02 \\
3.672 \mathrm{E}+01 \\
6.943 \mathrm{E}+00 \\
9.894 \mathrm{E}-01 \\
6.526 \mathrm{E}-02 \\
4.553 \mathrm{E}-04 \\
0.000 \mathrm{E}+00 \\
0.000 \mathrm{E}+00 \\
0.000 \mathrm{E}+00 \\
0.000 \mathrm{E}+00 \\
0.000 \mathrm{E}+00 \\
0.000 \mathrm{E}+00\end{array}$ & $\begin{array}{l}3.819 \mathrm{E}+09 \\
2.131 \mathrm{E}+08 \\
9.770 \mathrm{E}+06 \\
7.992 \mathrm{E}+06 \\
3.170 \mathrm{E}+09 \\
1.776 \mathrm{E}+05 \\
9.894 \mathrm{E}-01 \\
6.526 \mathrm{E}-02 \\
1.865 \mathrm{E}+06 \\
2.486 \mathrm{E}+06 \\
7.637 \mathrm{E}+04 \\
1.154 \mathrm{E}+05 \\
6.926 \mathrm{E}+03 \\
6.127 \mathrm{E}+04 \\
1.954 \mathrm{E}+03\end{array}$ & $\begin{array}{ll}16 & 0 . \\
17 & 0 . \\
18 & 0 . \\
19 & 0 . \\
20 & 0 . \\
21 & 0 . \\
22 & 0 . \\
23 & 0 . \\
24 & 0 . \\
25 & 0 . \\
26 & 0 . \\
27 & 0 . \\
28 & 0 . \\
29 & 0 . \\
30 & 0 .\end{array}$ & $\begin{array}{l}000 \mathrm{E}+00 \\
000 \mathrm{E}+00 \\
000 \mathrm{E}+00 \\
000 \mathrm{E}+00 \\
000 \mathrm{E}+00 \\
000 \mathrm{E}+00 \\
000 \mathrm{E}+00 \\
000 \mathrm{E}+00 \\
000 \mathrm{E}+00 \\
000 \mathrm{E}+00 \\
000 \mathrm{E}+00 \\
000 \mathrm{E}+00 \\
000 \mathrm{E}+00 \\
000 \mathrm{E}+00 \\
000 \mathrm{E}+00\end{array}$ & $\begin{array}{l}0.000 E+00 \\
0.000 E+00 \\
0.000 E+00 \\
0.000 E+00 \\
0.000 E+00 \\
0.000 E+00 \\
0.000 E+00 \\
0.000 E+00 \\
0.000 E+00 \\
0.000 E+00 \\
0.000 E+00 \\
0.000 E+00 \\
0.000 E+00 \\
0.000 E+00 \\
0.000 E+00\end{array}$ & $\begin{array}{l}0.000 \mathrm{E}+00 \\
0.000 \mathrm{E}+00 \\
0.000 \mathrm{E}+00 \\
0.000 \mathrm{E}+00 \\
0.000 \mathrm{E}+00 \\
0.000 \mathrm{E}+00 \\
0.000 \mathrm{E}+00 \\
0.000 \mathrm{E}+00 \\
0.000 \mathrm{E}+00 \\
0.000 \mathrm{E}+00 \\
0.000 \mathrm{E}+00 \\
0.000 \mathrm{E}+00 \\
0.000 \mathrm{E}+00 \\
0.000 \mathrm{E}+00 \\
0.000 \mathrm{E}+00\end{array}$ \\
\hline
\end{tabular}


WHC-SD-WM-CN-024

Rev. 0

Page 32 of 36

$L-3$

$\begin{array}{ccl}\text { Source } & \text { Shields } & \text { Distance to Detector, } X=9.910 E+01 \mathrm{~cm} \\ \text { Spherical } & \text { Spherical } & \text { Source Volume }=3.054 E+00 \mathrm{cc}\end{array}$

Source Mass $=3.054 E+00$ grams

Shield Thickness: $9.000 \mathrm{E}-01,9.820 \mathrm{E}+01 \mathrm{~cm}$

Distances from Dose Point to the Outside of

(1) Source Region: $9.820 \mathrm{E}+01 \mathrm{~cm}$ (2) Next Layer: $1.550 \mathrm{E}-06 \mathrm{~cm}$

Dose Buildup Data for Shield 1 with Effective Atomic Number 10.78

Fluence-to-Dose Conversion: Frontal (AP)

\begin{tabular}{|c|c|c|c|c|}
\hline $\begin{array}{l}\text { Average } \\
\mathrm{E} \text {, MeV }\end{array}$ & $\begin{array}{r}\text { Source Total } \\
\text { photons/sec }\end{array}$ & $\begin{array}{l}\text { Fluence to } \\
\text { Dose Factor }\end{array}$ & $\begin{array}{l}\text { Energy Fluence } \\
\mathrm{MeV} / \mathrm{sq} . \mathrm{cm} / \mathrm{sec}\end{array}$ & $\begin{array}{c}\text { Dose Rate } \\
\text { rem/hr }\end{array}$ \\
\hline $\begin{array}{l}0.015 \\
0.025 \\
0.035 \\
0.045 \\
0.055 \\
0.065 \\
0.075 \\
0.085 \\
0.095 \\
0.150 \\
0.250 \\
0.350 \\
0.475 \\
0.650 \\
0.825 \\
1.000 \\
1.225 \\
1.475 \\
1.700 \\
1.900 \\
2.100 \\
2.300\end{array}$ & $\begin{array}{l}5.030 \mathrm{E}+09 \\
8.224 \mathrm{E}+08 \\
6.959 \mathrm{E}+09 \\
2.337 \mathrm{E}+08 \\
3.336 \mathrm{E}+09 \\
1.308 \mathrm{E}+08 \\
1.118 \mathrm{E}+08 \\
9.470 \mathrm{E}+07 \\
8.345 \mathrm{E}+07 \\
3.900 \mathrm{E}+08 \\
1.094 \mathrm{E}+08 \\
4.944 \mathrm{E}+07 \\
3.602 \mathrm{E}+07 \\
8.159 \mathrm{E}+10 \\
7.410 \mathrm{E}+06 \\
4.947 \mathrm{E}+06 \\
7.724 \mathrm{E}+07 \\
8.119 \mathrm{E}+05 \\
7.062 \mathrm{E}+05 \\
3.951 \mathrm{E}+04 \\
2.486 \mathrm{E}+04 \\
4.737 \mathrm{E}+01\end{array}$ & $\begin{array}{l}3.871 \mathrm{E}-06 \\
4.148 \mathrm{E}-06 \\
3.518 \mathrm{E}-06 \\
2.979 \mathrm{E}-06 \\
2.598 \mathrm{E}-06 \\
2.336 \mathrm{E}-06 \\
2.155 \mathrm{E}-06 \\
2.028 \mathrm{E}-06 \\
1.940 \mathrm{E}-06 \\
1.801 \mathrm{E}-06 \\
1.870 \mathrm{E}-06 \\
1.870 \mathrm{E}-06 \\
1.838 \mathrm{E}-06 \\
1.775 \mathrm{E}-06 \\
1.709 \mathrm{E}-06 \\
1.647 \mathrm{E}-06 \\
1.575 \mathrm{E}-06 \\
1.505 \mathrm{E}-06 \\
1.449 \mathrm{E}-06 \\
1.405 \mathrm{E}-06 \\
1.366 \mathrm{E}-06 \\
1.330 \mathrm{E}-06\end{array}$ & $\begin{array}{l}5.684 \mathrm{E}+01 \\
7.015 \mathrm{E}+01 \\
1.426 \mathrm{E}+03 \\
7.686 \mathrm{E}+01 \\
1.482 \mathrm{E}+03 \\
7.147 \mathrm{E}+01 \\
7.226 \mathrm{E}+01 \\
7.068 \mathrm{E}+01 \\
7.072 \mathrm{E}+01 \\
5.079 \mathrm{E}+02 \\
2.388 \mathrm{E}+02 \\
1.493 \mathrm{E}+02 \\
1.447 \mathrm{E}+02 \\
4.408 \mathrm{E}+05 \\
5.035 \mathrm{E}+01 \\
4.050 \mathrm{E}+01 \\
7.700 \mathrm{E}+02 \\
9.705 \mathrm{E}+00 \\
9.708 \mathrm{E}+00 \\
6.061 \mathrm{E}-01 \\
4.210 \mathrm{E}-01 \\
8.779 \mathrm{E}-04\end{array}$ & $\begin{array}{l}2.200 \mathrm{E}-04 \\
2.909 \mathrm{E}-04 \\
5.015 \mathrm{E}-03 \\
2.289 \mathrm{E}-04 \\
3.849 \mathrm{E}-03 \\
1.670 \mathrm{E}-04 \\
1.557 \mathrm{E}-04 \\
1.433 \mathrm{E}-04 \\
1.372 \mathrm{E}-04 \\
9.146 \mathrm{E}-04 \\
4.467 \mathrm{E}-04 \\
2.793 \mathrm{E}-04 \\
2.660 \mathrm{E}-04 \\
7.824 \mathrm{E}-01 \\
8.606 \mathrm{E}-05 \\
6.671 \mathrm{E}-05 \\
1.213 \mathrm{E}-03 \\
1.460 \mathrm{E}-05 \\
1.407 \mathrm{E}-05 \\
8.518 \mathrm{E}-07 \\
5.750 \mathrm{E}-07 \\
1.168 \mathrm{E}-09\end{array}$ \\
\hline Totals: & $9.906 \mathrm{E}+10$ & tons/sec & $4.461 E+05$ & $\begin{array}{l}7.959 \mathrm{E}-01 \\
7.959 \mathrm{E}-03\end{array}$ \\
\hline
\end{tabular}

Closing: DAT'S ALL PHOLQUES!!!!!!!!

Finish run at $15: 51: 07 \quad 03 / 27 / 96$ 
WHC-SD-WM-CN-024

Rev. 0

Page 33 of 36

Input File (D:\ISOSHLD INPUT\IX_COLUMN.SRC) is shown below:

0

$L-3$

2 IX Column Transfer Box

\&INPUT

TEXT $=1, \quad I G E O M=3, N S H L D=1, J B U F=1$, DUNIT $=1$,

$T(1)=.90, X=99.1$, IPRNT $=0$, OPTION=1, ISPEC $=3$,

WEIGHT $(496)=2.40 \mathrm{E}-01$, WEIGHT $(490)=2.32 \mathrm{E}-06$, WEIGHT $(520)=1.51 \mathrm{E}-05$,

WEIGHT (476) $=6.69 \mathrm{E}-07$, WEIGHT (526) $=1.55 \mathrm{E}-05$, WEIGHT $(492)=4.25 \mathrm{E}-03$,

WEIGHT (493) $=4.91 \mathrm{E}-02$, WEIGHT (494) $=2.22 \mathrm{E}-02$, WEIGHT $(495)=4.53 \mathrm{E}-01$,

WEIGHT $(497)=6.37 \mathrm{E}-06$, WEIGHT (335) $=2.58 \mathrm{E}+00$, WEIGHT $(336)=2.45 \mathrm{E}+00$,

WEIGHT (082) $=4.17 \mathrm{E}-01$, WEIGHT $(084)=4.17 \mathrm{E}-01$, WEIGHT $(472)=1.01 \mathrm{E}-03$, WEIGHT (502) $=2.32 \mathrm{E}-06$, \&

1 SOURCE21 1.00

DAT'S ALL PHOLQUES!!!!!!!!

\&INPUT NEXT $=6$, \& 
WHC-SD-WM-CN-024

Rev. 0

Page 34 of 36

\section{CHECKLIST FOR INDEPENDENT TECHNICAL REVIEW}

DOCUMENT REVIEWED

NUMBER: WHC-SD-WM-CN-024

TITLE: CALCULATIONAL NOTE IN SUPPORT OF INTERNAL MEMO 8M730-HJG-96-007

Reviewer(s): P. D. Rittmann

I. Method(s) of Review

() Input data checked for accuracy
Independent calculation performed
(Hand calculation
() Alternate computer code:
() Alternate method (define)

II. Checklist (either check or enter NA if not applied)

( ) Task completely defined

( ) Activity consistent with task specification

() Necessary assumptions explicitly stated and supported

( ) Resources properly identified and referenced

( ) Resource documentation appropriate for this application

( Input data explicitly stated

( Input data verified to be consistent with original source

( ) Geometric model adequate representation of actual geometry

() Material properties appropriate and reasonable

( ) Mathematical derivations checked including dimensional consistency

() Hand calculations checked for errors

( ) Assumptions explicitly stated and justified

( ) Computer software appropriate for task and used within range of validity

() Use of resource outside range of established validity is justified

( ) Software runstreams correct and consistent with results

() Software output consistent with input

() Results consistent with applicable previous experimental or analytical findings

() Results and conclusions address all points and are consistent with task requirements and/or established limits or criteria

( ) Conclusions consistent with analytical results and established limits

( ) Uncertainty assessment appropriate and reasonable

() Other (define)

III. Comments:

IV. REVIEWER: 


\section{CHECKLIST FOR INDEPENDENT TECHNICAL REVIEW}

DOCUMENT REVIEWED

NUMBER: WHC-SD-WM-CN-024

TITLE: CALCULATIONAL NOTE IN SUPPORT OF INTERNAL MEMO 8M730-HJG-96-007

Reviewer $(s): D$. E. Lessor

I. Method(s) of Review

( ) Input data checked for accuracy

() Independent calculation performed

() Hand calculation

( ) Alternate computer code:

( ) Comparison to experiment or previous results

() Alternate method (define)

II. Checklist (either check or enter NA if not applied)

( ) Task completely defined

() Activity consistent with task specification

( ) Necessary assumptions explicitly stated and supported

() Resources properly identified and referenced

() Resource documentation appropriate for this application

() Input data explicitly stated

( ) Input data verified to be consistent with original source

() Geometric model adequate representation of actual geometry

( Material properties appropriate and reasonable

() Mathematical derivations checked including dimensional consistency

( ) Hand calculations checked for errors

( ) Assumptions explicitly stated and justified

( ) Computer software appropriate for task and used within range of validity

( ) Use of resource outside range of established validity is justified

( ) Software runstreams correct and consistent with results

() Software output consistent with input

() Results consistent with applicable previous experimental or analytical findings

() Results and conclusions address all points and are consistent with task requirements and/or established limits or criteria

( ) Conclusions consistent with analytical results and established limits

() Uncertainty assessment appropriate and reasonable

() Other (define)

III. Comments:

IV. REVIEWER: 
WHC-SD-WM-CN-024

Rev. 0

Page 36 of 36

\section{HEDOP REVIEW CHECKLIST \\ for \\ Radiological and Nonradiological Release Calculations}

Document Reviewed:

CALCULATIONAL NOTE IN SUPPORT OF INTERNAL MEMO 8M730-HJG-96-007

Submitted by: H. J. Goldberg Date Submitted: May 21996

Scope of Review: GXQ and GENII Calculations

YES NO* N/A

[ ] [ ] [ ] 1. A detailed technical review and approval of the environmental transport and dose calculation portion of the analysis has been performed and documented.

[ ] [ ] [ ] 2. Detailed technical review(s) and approval(s) of scenario and release determinations have been performed and documented.

[ ] [ ] [ ] 3. HEDOP-approved code(s) were used.

4. Receptor locations were selected according to HEDOP recommendations.

5. A11 applicable environmental pathways and code options were included and are appropriate for the calculations.

[ ] [ ] [ ] 6. Hanford site data were used.

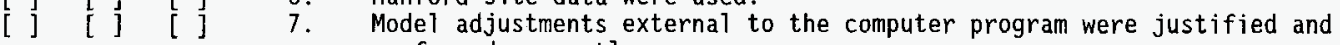
performed correctly.

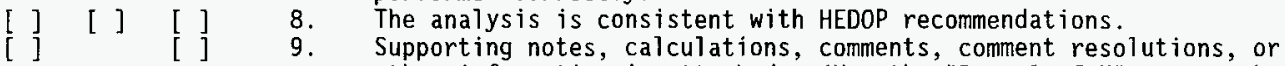
other information is attached. (Use the "Page 1 of $X$ " page numbering format and sign and date each added page.)

[ ] [ ] 10. Approval is granted on behalf of the Hanford Environmental Dose Overview Panel.

* A11 "NO" responses must be explained and use of nonstandard methods justified.

Reviewer Name: Paul D. Rittmann, B.A., M.S., Ph.D., C.H.P.

(print or type) 
DISTRIBUTION SHEET

\begin{tabular}{|c|c|c|c|c|c|}
\hline \multirow{2}{*}{$\begin{array}{l}\text { To } \\
\text { Distribution }\end{array}$} & \multirow{2}{*}{\multicolumn{3}{|c|}{$\begin{array}{l}\text { From } \\
\text { Criticality and Shielding }\end{array}$}} & \multicolumn{2}{|l|}{ Page 1 of 1} \\
\hline & & & & \multicolumn{2}{|c|}{ Date June 26,1996} \\
\hline \multicolumn{4}{|l|}{ Project Title/Work Order } & \multicolumn{2}{|c|}{ EDT No. 616558} \\
\hline \multicolumn{4}{|c|}{$\begin{array}{l}\text { Dose Consequence Analys is for Transporting Plutonium Recycle } \\
\text { Test Reactor (PRTR) Rupture Loop Ion Exchange Columns }\end{array}$} & \multicolumn{2}{|l|}{ ECN No. } \\
\hline Name & MSIN & $\begin{array}{l}\text { Text } \\
\text { With All } \\
\text { Attach. }\end{array}$ & Text Only & $\begin{array}{l}\text { Attach./ } \\
\text { Appendix } \\
\text { Only }\end{array}$ & $\begin{array}{l}\text { EDT/ECN } \\
\text { Only }\end{array}$ \\
\hline
\end{tabular}

J. G. Field

H. J. Goldberg

J. R. Green

J. Greenborg/File (2)

A. L. Ramble

Central Files (Original $+\mathrm{T}$ )

G1-11 $X$

HO-35 $X$

G1-11 $X$

HO-35 $X$

A3-38 $X$

n3-88 $X$ 